\title{
Laws of Large Numbers for Non-Homogeneous Markov Systems
}

\author{
P.-C. G. Vassiliou ${ }^{1}$
}

Received: 4 August 2017 / Revised: 27 November 2017 /

Accepted: 10 December 2017 / Published online: 4 January 2018

(C) The Author(s) 2018. This article is an open access publication

\begin{abstract}
In the present we establish Laws of Large Numbers for Non-Homogeneous Markov Systems and Cyclic Non-homogeneous Markov systems. We start with a theorem, where we establish, that for a NHMS under certain conditions, the fraction of time that a membership is in a certain state, asymptotically converges in mean square to the limit of the relative population structure of memberships in that state. We continue by proving a theorem which provides the conditions under which the mode of covergence is almost surely. We continue by proving under which conditions a Cyclic NHMS is Cesaro strongly ergodic. We then proceed to prove, that for a Cyclic NHMS under certain conditions the fraction of time that a membership is in a certain state, asymptotically converges in mean square to the limit of the relative population structure in the strongly Cesaro sense of memberships in that state. We then proceed to establish a founding Theorem, which provides the conditions under which, the relative population structure asymptotically converges in the strongly Cesaro sense with geometrical rate. This theorem is the basic instrument missing to prove, under what conditions the Law of Large Numbers for a Cycl-NHMS is with almost surely mode of convergence. Finally, we present two applications firstly for geriatric and stroke patients in a hospital and secondly for the population of students in a University system.
\end{abstract}

Keywords Non-homogeneous Markov systems · Cyclic non homogeneous Markov systems · Laws of large numbers

Mathematics Subject Classification (2010) $60 \mathrm{~J} 10 \cdot 60 \mathrm{~J} 20$

P.-C. G. Vassiliou

vasiliou@math.auth.gr

1 Department of Statistical Sciences, University College London, Gower Street,

London WC1E 6BT, UK 


\section{Introductory Notes}

One of the most celebrated theorems in probability theory is the Law of Large Numbers (Grimmett and Stirzaker 2001). The Law of Large Numbers were also studied for finite Markov chains (Kemeny and Snell 1981). The Law of Large Numbers for a regular homogeneous Markov chain states, that if $\pi_{j}$ is the limiting probability of being in state $j$ independent of the initial state, then also $\pi_{j}$ represents the fraction of time, that the process can be expected to be in state $j$ for a large number of steps. The Law of Large Numbers for Markov chains is also linked with the Martingale Convergence Theorem (Kemeny et al. 1976). Laws of Large Numbers were also studied for non-homogeneous semi-Markov processes by Vadori and Swishchuk (2015). For Markov chains in general state spaces there exists a chapter on Laws of Large Numbers in Meyn and Tweedie (2009), where the theory of martingales is the main instrument for proving various types of LLN. These laws are of value for Markov chains exactly as they are for all stochastic processes: the LLN and CLT, in particular, provide the theoretical basis for many results in the statistical analysis of chains as they do in related fields. For this and other applications, the reader is referred to Hall and Heyde (1980).

In the present paper we will study the Laws of Large Numbers for Non-Homogeneous Markov Systems and for Cyclic Non-Homogeneous Markov systems. The theory of NHMSs has its roots in the use of Markov models in manpower systems, which started with the work of Young and Almond (1961) and Bartholomew (1963). Young's motive was the application of homogeneous Markov chain models in the British University system. Bartholomew created important multiple renewal theory models for various social processes and his first related book Bartholomew (1967) among other things, provided an important theoretical reference of applied probability style for everyone. The concept of Non-homogeneous Markov systems was first introduced in Vassiliou (1982). From then onwards a vast literature in a great variety of journals was created by many authors, a sample of which could be found in the review papers by Vassiliou (1997) and Ugwuowo and McClean (2000). The motive was to provide a more general framework for a number of Non-homogeneous Markov chain models in manpower systems. There is also a great variety of applied probability models, that could be accommodated in this general framework. Let us consider a population (system), which is stratified into classes (states) according to various characteristics. The members of the system could be sections of human societies, parts of the animal kingdom, populations of fisheries, biological micro-organisms, particles in a physical phenomenon, various types of machines, various types of cells or viruses of the human body etc. The members of the system are categorized into various states, according to the problem at hand. The set of states are assumed to be exclusive, so that each member of the system may be in one and only one state at a given time. We call population structure, the vector containing the number of members of each state in the system. Members are leaving the system in a stochastic way and also new members are entering the system in a stochastic way. In fact a non-homogeneous Markov chain is a NHMS with one particle as a member, which never leaves the system and in which no other particles enter.

There are a large number of applications of the theory of NHMS and in quite diverse areas, where the present results will have an impact. We will only refer to some of these applications that contribute to the health care of human beings. For example, applications to the evolution of the population of HIV virus within the human of T-cells in Mathiew et al. (2006), and Foucher et al. (2005); gene expression sequences in McClean et al. (2003); in hospital and geriatric patient care McClean et al. (1998a, b), Taylor et al. (2000), Faddy and McClean (2005), Garg et al. (2010), McClean and Millard (2007), Marshall et al. (2002), 
Marshall and McClean (2003, 2004). Garg et al. (2009), Lalit et al. (2010), McClean et al. (2014a) and McClean et al. (2014b).

The paper is organized as follows: In Section 2 we provide basic concepts and useful results for a NHMS, which are known or slightly amended. Also we provide some useful in what follows theorems on the various modes of convergence of random variables in a probability space. The results in this section will be used repeatedly in the sections that follow. In Section 3 we first prove a theorem which is a Law of Large Numbers for a NHMS. We prove, that for a NHMS under certain conditions the fraction of time that a membership is in a certain state, asymptotically converges in mean square to the limit of the relative population structure of membership in that state. In a second theorem in the same section we provide and prove under what conditions the mode of convergence in the previous basic result is almost surely. In Section 4 we study the important category of Cyclic NHMS, a concept which was motivated by the work of Gani (1963) on students enrolment at Michigan state University and Bartholomew (1982). We prove in two theorems, under what conditions the relative population structure of a Cycl-NHMS asymptotically converges in the strongly Cesaro sense. In Section 5 we first prove a theorem which is a Law of Large Numbers for a Cycl-NHMS. We prove, that for a Cycl- NHMS under certain conditions the fraction of time that a membership is in a certain state, asymptotically converges in mean square to the limit of the relative population structure in the strongly Cesaro sense of membership in that state. We then proceed to establish a founding Theorem, which provides the conditions under which the relative population structure asymptotically converges in the strongly Cesaro sense with geometrical rate. This theorem is the basic instrument missing to prove, under what conditions the Law of Large Numbers for a Cycl-NHMS is with almost surely mode of convergence. In Section 6 we provide applications of the present results in Section 3 to geriatric and stroke patients. Also, we provide applications of the results in Sections 4 and 5 for the movements of students in a University system.

\section{Basic Concepts and Useful Results for a NHMS in Discrete Time}

We firstly recall the concept of an NHMS and introduce concepts and known results necessary for the study of the Law of Large numbers for NHMSs. Consider a population (system) which is stratified into classes (states) according to various characteristics. Let $S=\{1,2, \ldots, k\}$ be the set of states, that are assumed to be exclusive and exhaustive. Let, that we have a discrete time scale $t=0,1,2, \ldots$ and $\{\mathbf{P}(t)\}_{t=0}^{\infty}$ be the sequence of transition probability matrices between the states. Assume, that we have wastage from the system and denote by $\omega$ the state which represents the external environment of the system to which the population members, who leave the system go. Let $\left\{\mathbf{p}_{\omega}(t)\right\}_{t=0}^{\infty}$ be the vector of probabilities of wastage from the various states of the system. Let $\{T(t)\}_{t=0}^{\infty}$ be the total number of memberships of the system at time $t$, which is assumed to be a realization of a known stochastic process. We assume that each member holds a membership, which is left, when the member leaves the system and is taken by new members entering the system to replace leavers or to expand the system. Apparently, $T(t) \geq 0$ and it is assumed that $\Delta T(t)=T(t+1)-T(t) \geq 0$. Let $\left\{\mathbf{p}_{0}(t)\right\}_{t=0}^{\infty}$ be the vector of probabilities of allocation of replacements and new memberships, in the various states of the system, which is being done independently of internal movements. Denote by $\mathbf{Q}(t)=\mathbf{P}(t)+\mathbf{p}_{\omega}^{\top}(t) \mathbf{p}_{0}(t)$; then $\mathbf{Q}(t)$ is a stochastic matrix, and the non-homogeneous Markov chain defined by the sequence $\{\mathbf{Q}(t)\}_{t=0}^{\infty}$ will be called the imbedded non-homogeneous Markov chain of the NHMS. Define by $N_{i}(t)$ the random variable representing the number of memberships in 
state $i$ at time $t ; \mathbf{N}(t)=\left[N_{1}(t), N_{2}(t), \ldots, N_{k}(t)\right]$ the vector of the random variables representing the population structure of the NHMS. Let $\mathbf{q}(t)=\mathbf{N}(t) / T(t)$ be the relative population structure. Define by

$$
\mathbf{q}(s, t)=\left[q_{1}(s, t), q_{2}(s, t), \ldots, q_{k}(s, t)\right],
$$

where

$$
q_{j}(s, t)=\mathbb{P}\left[X_{t}=j \mid \mathbf{q}(s)\right], \quad \text { for } s \leq t,
$$

then from Georgiou and Vassiliou (1992) p.140 we get that

$$
\mathbb{E}[\mathbf{q}(t-1, t)]=a(t-1) \mathbf{q}(t-1) \mathbf{Q}(t-1)+b(t-1) \mathbf{p}_{0}(t-1),
$$

where

$$
a(t-1)=\frac{T(t-1)}{T(t)} \text { and } b(t-1)=\frac{T(t)-T(t-1)}{T(t)} .
$$

also we get that

$$
\mathbb{E}[\mathbf{q}(0, t)]=a(t-1) \mathbb{E}[\mathbf{q}(0, t-1)] \mathbf{Q}(t-1)+b(t-1) \mathbf{p}_{0}(t-1),
$$

from which recursively we get (see Georgiou and Vassiliou (1992) p.149) that

$$
\begin{aligned}
\mathbb{E}[\mathbf{q}(0, t)]= & \frac{T(0)}{T(t)} \mathbf{q}(0) \mathbf{Q}(0, t-1) \\
& +\frac{1}{T(t)} \sum_{\tau=1}^{t} \Delta T(\tau-1) \mathbf{p}_{0}(\tau-1) \mathbf{Q}(\tau, t-1),
\end{aligned}
$$

where $\mathbf{Q}(s, t)=\mathbf{Q}(s) \mathbf{Q}(s+1) \ldots \mathbf{Q}(t)$ for $s \leq t$. We set $\mathbf{Q}(s, t)=\mathbf{I}$ the identity matrix for $s>t$. Note also that we set $\mathbf{q}(s, t)=\mathbf{0}$ for $s>t$.

We denote by

$$
\mathbb{E}[\mathbf{q}(s, t)]=\left[\mathbb{E}\left[q_{1}(s, t)\right], \mathbb{E}\left[q_{2}(s, t)\right], \ldots, \mathbb{E}\left[q_{k}(s, t)\right]\right],
$$

and apparently we have

$$
\begin{aligned}
\mathbb{E}[\mathbf{q}(s, t)]= & \frac{T(s)}{T(t)} \mathbf{q}(s) \mathbf{Q}(s, t-1) \\
& +\frac{1}{T(t)} \sum_{\tau=s+1}^{t} \Delta T(\tau-1) \mathbf{p}_{0}(\tau-1) \mathbf{Q}(\tau, t-1) .
\end{aligned}
$$

We denote by $\mathbb{E}\left[\mathbf{q}^{(i)}(s, t)\right]$ if it is known that

$$
\mathbf{q}(s)=[0,0, \ldots, \underbrace{1}_{i-t h}, \ldots, 0],
$$

and the vector $\mathbb{E}\left[\mathbf{q}^{(i)}(s, t)\right]$ is then

$$
\mathbb{E}\left[\mathbf{q}^{(i)}(s, t)\right]=\left[\mathbb{E}\left[q_{1}^{(i)}(s, t)\right], \mathbb{E}\left[q_{2}^{(i)}(s, t)\right], \ldots, \mathbb{E}\left[q_{k}^{(i)}(s, t)\right]\right] .
$$

Let $\mathcal{M}_{n, m}(\mathbb{R})$ be the vector space of all $n \times m$ real matrices $\mathcal{S} \mathcal{M}_{n, n}$; the vector space of all $n \times n$ stochastic matrices. Let $\mathbf{Q} \in \mathcal{S} \mathcal{M}_{n, n}$, then it is regular if it's states consist of a single communicating class which is aperiodic or equivalently $\mathbf{Q}$ has 1 as the only eigenvalue with modulus 1 and with geometric multiplicity one. For $\mathbf{A} \in \mathcal{M}_{n, n}(\mathbb{R})$ we define the norm $\|$.

$$
\|\mathbf{A}\|=\sup _{i \in S} \sum_{j \in S}\left|a_{i j}\right| .
$$


Now from Vassiliou (1981) we get the following theorem:

Theorem 1 Let a NHMS and let that

a) $\lim _{t \rightarrow \infty}\|\mathbf{Q}(t)-\mathbf{Q}\|=0$ and $\mathbf{Q}$ a regular stochastic matrix;

b) $\lim _{t \rightarrow \infty}\left\|\mathbf{p}_{0}(t)-\mathbf{p}_{0}\right\|=0$;

c) $\lim _{t \rightarrow \infty}[\Delta T(t) / T(t)]=0$,

then

$$
\lim _{t \rightarrow \infty}\|\mathbb{E}[\mathbf{q}(0, t)]-\mathbf{q}(\infty)\|=0,
$$

where $\mathbf{q}(\infty)$ is the row of the stable stochastic matrix $\mathbf{Q}(\infty)=\lim _{t \rightarrow \infty} \mathbf{Q}^{t}$.

From Isaacson and Madsen p.157 and p.170 we get the following two theorems

Theorem 2 A non-homogeneous Markov chain with transition matrices $\{\mathbf{Q}(t)\}_{t=0}^{\infty}$ is strongly ergodic if and only if there exists a constant matrix $\mathbf{Q}$ such that for each $m$

$$
\lim _{t \rightarrow \infty}\|\mathbf{Q}(m, t)-\mathbf{Q}\|=0 .
$$

Theorem 3 Let $\{\mathbf{Q}(t)\}_{t=0}^{\infty}$ be a sequence of transition matrices corresponding to a nonhomogeneous Markov chain. If $\lim _{t \rightarrow \infty}\|\mathbf{Q}(t)-\mathbf{Q}\|=0$ where $\mathbf{Q}$ is weakly ergodic, then the chain is strongly ergodic.

Following the steps of the proof of Theorem 1 in Vassiliou (1981) and using Theorems 2 and 3 we arrive at

\section{Theorem 4 Let a NHMS and let that}

a) $\lim _{t \rightarrow \infty}\|\mathbf{Q}(t)-\mathbf{Q}\|=0$ and $\mathbf{Q}$ a regular stochastic matrix;

b) $\lim _{t \rightarrow \infty}\left\|\mathbf{p}_{0}(t)-\mathbf{p}_{0}\right\|=0$;

c) $\lim _{t \rightarrow \infty}[\Delta T(t) / T(t)]=0$,

then

$$
\lim _{t \rightarrow \infty}\|\mathbb{E}[\mathbf{q}(s, t)]-\mathbf{q}(\infty)\|=0, \text { for every } s \leq t .
$$

Let a probability space $(\Omega, \mathcal{F}, \mathbb{P})$ and a sequence of random variables $\left\{X_{n}\right\}_{n=0}^{\infty}$ with $X_{n}: \Omega \rightarrow \mathbb{R}$. It is well known that there are various modes of convergence of the sequence $\left\{X_{n}\right\}_{n=0}^{\infty}$ to a random variable $X: \Omega \rightarrow \mathbb{R}$. We now provide the formal definition of three of these modes.

Definition 1 Let a probability space $(\Omega, \mathcal{F}, \mathbb{P})$ and a sequence of random variables $\left\{X_{n}\right\}_{n=0}^{\infty}$ with $X_{n}: \Omega \rightarrow \mathbb{R}$ and a random variable $X: \Omega \rightarrow \mathbb{R}$. We say that the sequence of random variables $\left\{X_{n}\right\}_{n=0}^{\infty}$ converge almost surely to the random variable $X$ if the event

$$
\left\{\omega \in \Omega: X_{n}(\omega) \rightarrow X(\omega) \text { as } n \rightarrow \infty\right\},
$$

has probability one. We will denote this type of convergence by

$$
X_{n} \stackrel{\text { a.s. }}{\rightarrow} X \text { or } \lim _{n \rightarrow \infty} X_{n}=X \text { a.s. }
$$

Definition 2 Let a probability space $(\Omega, \mathcal{F}, \mathbb{P})$ and a sequence of random variables $\left\{X_{n}\right\}_{n=0}^{\infty}$ with $X_{n}: \Omega \rightarrow \mathbb{R}$ and a random variable $X: \Omega \rightarrow \mathbb{R}$. If $\left|X_{n}\right|$ and $|X|$ are in $L^{p}$ 
where $1 \leq p \leq \infty$, i.e., $\mathbb{E}\left[\left|X_{n}^{p}\right|\right]<\infty$ for all $n$ and $\mathbb{E}[|X|]<\infty$, then we say that the sequence of random variables $\left\{X_{n}\right\}_{n=0}^{\infty}$ converges to $X$ in $p$-th mean and we denote it by

$$
X_{n} \stackrel{L^{p}}{\rightarrow} X
$$

if and only if

$$
\lim _{n \rightarrow \infty} \mathbb{E}\left\{\left|X_{n}-X\right|^{p}\right\}=0
$$

One of the most useful modes of convergence is the mean square, that is, for $p=2$ we have

$$
X_{n} \stackrel{L^{2}}{\rightarrow} X, \text { or } X_{n} \rightarrow X \text { in mean square, or } X_{n} \stackrel{\text { m.s. }}{\rightarrow} X
$$

Definition 3 Let a probability space $(\Omega, \mathcal{F}, \mathbb{P})$ and a sequence of random variables $\left\{X_{n}\right\}_{n=0}^{\infty}$ with $X_{n}: \Omega \rightarrow \mathbb{R}$ and a random variable $X: \Omega \rightarrow \mathbb{R}$. We say that $X_{n} \rightarrow X$ in probability, and we write $X_{n} \stackrel{P}{\rightarrow} X$, if

$$
\mathbb{P}\left(\left|X_{n}-X\right|>\epsilon\right) \rightarrow 0 \text { as } n \rightarrow \infty \text { for all } \epsilon>0 .
$$

From Grimmett and Stirzaker (2001) p.311 we get the following Theorem.

Theorem 5 Let a probability space $(\Omega, \mathcal{F}, \mathbb{P})$ and a sequence of random variables $\left\{X_{n}\right\}_{n=0}^{\infty}$ with $X_{n}: \Omega \rightarrow \mathbb{R}$ and a random variable $X: \Omega \rightarrow \mathbb{R}$. Then (a) If $r>s \geq 1$ and $X_{n} \stackrel{L^{r}}{\rightarrow} X$ then $X_{n} \stackrel{L^{s}}{\rightarrow} X$. (b) If $X_{n} \stackrel{L^{1}}{\rightarrow} X$ then $X_{n} \stackrel{P}{\rightarrow} X$. The converse assertions fail in general.

Note that any sequence $\left\{X_{n}\right\}_{n=0}^{\infty}$ which satisfies $X_{n} \stackrel{P}{\rightarrow} X$ necessarily contains a subsequence $\left\{X_{n_{i}}: 1 \leq i<\infty\right\}$ which converge almost surely. From Grimmett and Stirzaker (2001) p.314 we get the following Theorem.

Theorem 6 Let a probability space $(\Omega, \mathcal{F}, \mathbb{P})$ and a sequence of random variables $\left\{X_{n}\right\}_{n=0}^{\infty}$ with $X_{n}: \Omega \rightarrow \mathbb{R}$ and a random variable $X: \Omega \rightarrow \mathbb{R}$. If $X_{n} \stackrel{P}{\rightarrow} X$, there exists $a$ non-random increasing sequence of integers $n_{1}, n_{2}, \ldots$ such that $X_{n_{i}} \stackrel{\text { a.s. }}{\rightarrow} X$ as $i \rightarrow \infty$.

Also from Grimmett and Stirzaker (2001) p.310 we get the following Theorem.

Theorem 7 Let a probability space $(\Omega, \mathcal{F}, \mathbb{P})$ and a sequence of random variables $\left\{X_{n}\right\}_{n=0}^{\infty}$ with $X_{n}: \Omega \rightarrow \mathbb{R}$ and a random variable $X: \Omega \rightarrow \mathbb{R}$. If $P_{n}(\epsilon)=\mathbb{P}\left(\left|X_{n}-X\right|>\epsilon\right)$ satisfies

$$
\sum_{n} P_{n}(\epsilon)<\infty \text { for all } \epsilon>0
$$

then $X_{n} \stackrel{\text { a.s. }}{\rightarrow} X$.

Theorem 8 (Chebychov inequality). Let a probability space $(\Omega, \mathcal{F}, \mathbb{P})$ and a random variable $X: \Omega \rightarrow \mathbb{R}$. Then

$$
\mathbb{P}(|X| \geq a) \leq \frac{\mathbb{E}\left(X^{2}\right)}{a^{2}} \text { if } a>0 .
$$

From Huang et al. (1976) we amend slightly the basic theorem to get that 
Theorem 9 Let a probability space $(\Omega, \mathcal{F}, \mathbb{P})$ and a non-homogeneous Markov chain be defined by the sequence of transition matrices $\{\mathbf{Q}(s, t)\}_{s, t}$. Let $\lim _{t \rightarrow \infty}\|\mathbf{Q}(t)-\mathbf{Q}\|=0$ geometrically fast with $\mathbf{Q}$ a regular stochastic matrix. Then $\|\mathbf{Q}(s, t)-\mathbf{Q}\|=0$ geometrically fast uniformly in $s$. That is, for every s there exists constants $c>0$ and $0<b<1$ such that

$$
\|\mathbf{Q}(s, t)-\mathbf{Q}\| \leq c b^{t-s} .
$$

From Vassiliou and Georgiou (1990) p.541 we get the following theorem:

Theorem 10 Let an NHMS be given with $\{\mathbf{P}(t)\}_{t=0}^{\infty},\{T(t)\}_{t=0}^{\infty},\left\{\mathbf{p}_{k+1}(t)\right\}_{t=0}^{\infty},\left\{\mathbf{p}_{0}(t)\right\}_{t=0}^{\infty}$. Assume that

$$
\text { a) } \left.\left.\lim _{t \rightarrow \infty}\|\mathbf{P}(t)-\mathbf{P}\|=0, b\right) \lim _{t \rightarrow \infty}\left\|\mathbf{p}_{k+1}(t)-\mathbf{p}_{k+1}\right\|=0, c\right)\left\|\mathbf{p}_{0}(t)-\mathbf{p}_{0}\right\|=0,
$$

the rate of convergence is geometric in all cases and $\mathbf{Q}=\mathbf{P}+\mathbf{p}_{\omega}^{\top} \mathbf{p}_{0}$ is regular. Also, $T(t) \geq$ $T(t-1)$ and

$$
\left\{\frac{\Delta T(t)}{T(t)}\right\}_{t=0}^{\infty} \text { converges to zero geometrically fast. }
$$

Then the sequence of relative structures converges to $\mathbf{q}(\infty)=\mathbf{p}_{0} \mathbf{Q}^{\infty}$ geometrically fast, where $\mathbf{Q}^{\infty}=\lim _{t \rightarrow \infty} \mathbf{Q}^{t}$.

\section{Laws of Large Numbers for a NHMS}

In the present section we will study the Law of Large Numbers for a NHMS. We will start with the mode of mean square convergence and then we will proceed to prove almost sure convergence. Let $X_{t}$ the random variable representing the state of a membership at time $t$. Define by

$$
u_{j}(t)=\left\{\begin{array}{lll}
1 & \text { if } & X_{t}=j \\
0 & \text { if } & X_{t} \neq j
\end{array}\right\}
$$

also let $y_{j}(t)$ be the random variable representing the number of times the membership is in state $j$ up to time $t$, i.e., $X_{s}=j, 1 \leq s \leq t ; v_{j}(t)$ be the random variable representing the fraction of time the membership is in state $j$ up to time $t$. We have that

$$
y_{j}(t)=\sum_{s=1}^{t} u_{j}(s) \quad \text { and } \quad v_{j}(t)=\frac{y_{j}(t)}{t} .
$$

Denote by

$$
\mathbf{u}(t)=\left[u_{1}(t), u_{2}(t), \ldots, u_{k}(t)\right], \mathbf{y}(t)=\left[y_{1}(t), y_{2}(t), \ldots, y_{k}(t)\right],
$$

and

$$
v(t)=\left[v_{1}(t), v_{2}(t), \ldots, v_{k}(t)\right] .
$$

We will now provide and prove the following theorem of the Law of Large Numbers for a NHMS

Theorem 11 Let a probability space $(\Omega, \mathcal{F}, \mathbb{P})$ and a NHMS be defined in Section 2. Assume that a) $\lim _{t \rightarrow \infty}\|\mathbf{Q}(t)-\mathbf{Q}\|=0$ and $\mathbf{Q}$ a regular stochastic matrix; $b$ ) $\left.\lim _{t \rightarrow \infty}\left\|\mathbf{p}_{0}(t)-\mathbf{p}_{0}\right\|=0 ; c\right) \lim _{t \rightarrow \infty}[\Delta T(t) / T(t)]=0$. Then

$$
v(t) \stackrel{L^{2}}{\rightarrow} \mathbf{q}(\infty) \text {. }
$$


Proof It is equivalent to show that

$$
\mathbb{E}\left[(v(t)-\mathbf{q}(\infty))^{2}\right]=0 .
$$

Since the dimension of the vectors is finite it is equivalent to show that

$$
\mathbb{E}\left[\left(v_{j}(t)-q_{j}(\infty)\right)^{2}\right]=0 \text { for every } j=1,2, \ldots, k .
$$

We have that

$$
\begin{aligned}
\mathbb{E}\left[\left(v_{j}(t)-q_{j}(\infty)\right)^{2}\right] & =\mathbb{E}\left(\sum_{n=1}^{t} \frac{u_{j}(n)}{t}-q_{j}(\infty)\right)^{2} \\
& =\frac{1}{t^{2}} \mathbb{E}\left(\sum_{n=1}^{t}\left(u_{j}(n)-q_{j}(\infty)\right)\right)^{2} \\
& =\frac{1}{t^{2}} \mathbb{E}\left[\left(\sum_{n=1}^{t}\left(u_{j}(n)-q_{j}(\infty)\right)\right)\left(\sum_{l=1}^{t}\left(u_{j}(l)-q_{j}(\infty)\right)\right)\right] .
\end{aligned}
$$

Hence we have that

$$
\begin{aligned}
\mathbb{E}\left[\left(v_{j}(t)-q_{j}(\infty)\right)^{2}\right]= & \frac{1}{t^{2}} \sum_{n=1}^{t} \sum_{l=1}^{t} \mathbb{E}\left[u_{j}(n) u_{j}(l)\right] \\
& -\frac{1}{t^{2}} \sum_{n=1}^{t} \sum_{l=1}^{t} q_{j}(\infty) \mathbb{E}\left[u_{j}(n)\right] \\
& -\frac{1}{t^{2}} \sum_{n=1}^{t} \sum_{l=1}^{t} q_{j}(\infty) \mathbb{E}\left[u_{j}(l)\right] \\
& +\frac{1}{t^{2}} \sum_{n=1}^{t} \sum_{l=1}^{t} q_{j}^{2}(\infty) .
\end{aligned}
$$

Now we have that

$$
\begin{aligned}
\lim _{t \rightarrow \infty}\left\{-\frac{1}{t^{2}} \sum_{n=1}^{t} \sum_{l=1}^{t} q_{j}(\infty) \mathbb{E}\left[u_{j}(n)\right]\right\} & =\lim _{t \rightarrow \infty}\left\{-\frac{1}{t^{2}} \sum_{n=1}^{t} \sum_{l=1}^{t} q_{j}(\infty) \mathbb{E}\left[\mathbb{E}\left[u_{j}(n) \mid \mathbf{q}(0)\right]\right]\right\} \\
& =\lim _{t \rightarrow \infty}\left\{-\frac{1}{t} q_{j}(\infty) \sum_{n=1}^{t} \mathbb{E}\left[\mathbb{P}\left(X_{n}=j \mid \mathbf{q}(0)\right)\right]\right\} \\
& =\lim _{t \rightarrow \infty}\left\{-q_{j}(\infty) \frac{1}{t} \sum_{n=1}^{t} \mathbb{E}\left[q_{j}(0, n)\right]\right\} \\
& =(\text { by Theorem } 1)=-q_{j}^{2}(\infty) .
\end{aligned}
$$

Similarly we get that

$$
\lim _{t \rightarrow \infty}\left\{-\frac{1}{t^{2}} \sum_{n=1}^{t} \sum_{l=1}^{t} q_{j}(\infty) \mathbb{E}\left[u_{j}(l)\right]\right\}=-q_{j}^{2}(\infty) .
$$


It is easy to see that

$$
\frac{1}{t^{2}} \sum_{n=1}^{t} \sum_{l=1}^{t} q_{j}^{2}(\infty)=q_{j}^{2}(\infty) .
$$

Finally it remains to find

$$
\begin{aligned}
\lim _{t \rightarrow \infty}\left\{\frac{1}{t^{2}} \sum_{n=1}^{t} \sum_{l=1}^{t} \mathbb{E}\left[u_{j}(n) u_{j}(l)\right]\right\} & =\lim _{t \rightarrow \infty}\left\{\frac{1}{t^{2}} \sum_{n=1}^{t} \sum_{l=1}^{t} \mathbb{E}\left[\mathbb{E}\left[u_{j}(n) u_{j}(l)\right] \mid \mathbf{q}(0)\right]\right\} \\
& =\lim _{t \rightarrow \infty}\left\{\frac{1}{t^{2}} \sum_{n=1}^{t} \sum_{l=1}^{t} \mathbb{E}\left[\mathbb{P}\left(X_{n}=j, X_{l}=j \mid \mathbf{q}(0)\right)\right]\right\} \\
& =A
\end{aligned}
$$

Define by $n \wedge l=\max \{n, l\}$ and $n \vee l=\min \{n, l\}$ then we have that

$$
\begin{aligned}
\mathbb{P}\left(X_{n \wedge l}=j, X_{n \vee l}=j \mid \mathbf{q}(0)\right) & =\mathbb{P}\left(X_{n \wedge l}=j \mid X_{n \vee l}=j, \mathbf{q}(0)\right) \mathbb{P}\left(X_{n \vee l}=j \mid \mathbf{q}(0)\right) \\
& =\mathbb{P}\left(X_{n \wedge l}=j \mid q_{j}(0, n \vee l)=1\right) \mathbb{P}\left(X_{n \vee l}=j \mid \mathbf{q}(0)\right) .
\end{aligned}
$$

Hence from Eqs. 3.13 and 3.14 we get that

$$
\begin{aligned}
A & =\lim _{t \rightarrow \infty}\left\{\frac{1}{t^{2}} \sum_{n=1}^{t} \sum_{l=1}^{t} \mathbb{E}\left[\mathbb{P}\left(X_{n}=j, X_{l}=j \mid \mathbf{q}(0)\right)\right]\right\} \\
& =\lim _{t \rightarrow \infty}\left\{\frac{1}{t^{2}} \sum_{n=1}^{t} \sum_{l=1}^{t} \mathbb{E}\left[\mathbb{P}\left(X_{n \wedge l}=j \mid q_{j}(0, n \vee l)=1\right)\right] \mathbb{E}\left[\mathbb{P}\left(X_{n \vee l}=j \mid \mathbf{q}(0)\right)\right]\right\} \\
& =\lim _{t \rightarrow \infty}\left\{\frac{1}{t^{2}} \sum_{n=1}^{t} \sum_{l=1}^{t} \mathbb{E}\left[q_{j}^{(j)}(n \vee l, n \wedge l)\right] \mathbb{E}\left[q_{j}(0, n \vee l)\right]\right\} \\
& =\left(\operatorname{since} q_{j}^{(j)}(s, t)=0 \text { for } s>t\right) \\
& =\lim _{t \rightarrow \infty}\left\{\frac{1}{t^{2}} \sum_{n=1}^{t} \sum_{l=n}^{t} \mathbb{E}\left[q_{j}^{(j)}(n, l)\right] \mathbb{E}\left[q_{j}(0, n)\right]\right\} \\
& =\lim _{t \rightarrow \infty}\left\{\frac{1}{t} \sum_{n=1}^{t} \mathbb{E}\left[q_{j}(0, n)\right] \frac{1}{t} \sum_{l=n}^{t} \mathbb{E}\left[q_{j}^{(j)}(n, l)\right]\right\}=(\text { by Theorems } 1,2 \text { and } 3) \\
& =q_{j}^{2}(\infty) .
\end{aligned}
$$

Hence from Eqs. 3.6, 3.7,..., 3.15 we get Eq. 3.4 which completes the proof.

Hence, we have actually proved, that under certain conditions the fraction of time the membership of an NHMS stays in a state after a large number of steps, converges in mean square to the limit of the relative population structure in that state. This result constitutes the Weak Law of Large Numbers for a NHMS. We are now going to proceed and prove under which conditions the mode of convergence is almost surely.

Theorem 12 Let a probability space $(\Omega, \mathcal{F}, \mathbb{P})$ and an NHMS as defined in Section 2. Assume that a) $\lim _{t \rightarrow \infty}\|\mathbf{Q}(t)-\mathbf{Q}\|=0$ geometrically fast and $\mathbf{Q}$ a regular stochastic matrix; $b) \lim _{t \rightarrow \infty}\left\|\mathbf{p}_{0}(t)-\mathbf{p}_{0}\right\|=0$ geometrically fast $\left.c\right) \lim _{t \rightarrow \infty}[\Delta T(t) / T(t)]=0$ geometrically fast. Then

$$
v(t) \stackrel{a . s .}{\rightarrow} \mathbf{q}(\infty) \text {. }
$$


Proof In Theorem 11 we have actually proved that

$$
v_{j}(t) \stackrel{L^{2}}{\rightarrow} q_{j}(\infty) \text { as } t \rightarrow \infty \text { for ever } j \in S,
$$

or equivalently

$$
\frac{1}{t} \sum_{s=1}^{t} u_{j}(s) \stackrel{L^{2}}{\rightarrow} q_{j}(\infty) \text { as } t \rightarrow \infty \text { for ever } j \in S,
$$

from Theorem 5 (a) and Eq. 3.17 we get that

$$
\frac{1}{t} \sum_{s=1}^{t} u_{j}(s) \stackrel{L^{1}}{\rightarrow} q_{j}(\infty) \text { as } t \rightarrow \infty \text { for ever } j \in S,
$$

from Theorem $5(b)$ and Eq. 3.18 we get that

$$
\frac{1}{t} \sum_{s=1}^{t} u_{j}(s) \stackrel{P}{\rightarrow} q_{j}(\infty) \text { as } t \rightarrow \infty \text { for ever } j \in S .
$$

By Theorem 6 there exists a non-random increasing sequence of integers $t_{1}, t_{2}, \ldots$ such that

$$
\frac{1}{t_{i}} \sum_{s=1}^{t_{i}} u_{j}(s) \stackrel{\text { a.s }}{\rightarrow} q_{j}(\infty) \text { as } i \rightarrow \infty \text { for ever } j \in S .
$$

We will now prove that such a choice of subsequence is $t_{i}=i^{2}$ for $i=1,2, \ldots$ In order to do so it is sufficient by Theorem 7 to show that

$$
\text { For every } \epsilon>0 \sum_{i} \mathbb{P}\left(\left|\frac{1}{i^{2}} \sum_{s=1}^{i^{2}} u_{j}(s)-q_{j}(\infty)\right|>\epsilon\right)<\infty .
$$

By Theorem 8 , that is, Chebychov inequality we get that

$$
\mathbb{P}\left(\left|\frac{1}{i^{2}} \sum_{s=1}^{i^{2}} u_{j}(s)-q_{j}(\infty)\right|>\epsilon\right) \leq \frac{\mathbb{E}\left[\left(\frac{1}{i^{2}} \sum_{s=1}^{i^{2}} u_{j}(s)-q_{j}(\infty)\right)^{2}\right]}{\epsilon^{2}} .
$$

Therefore, we should prove that

$$
\text { For every } \epsilon>0 B=\frac{1}{\epsilon^{2}} \sum_{i} \mathbb{E}\left[\left(\frac{1}{i^{2}} \sum_{s=1}^{i^{2}} u_{j}(s)-q_{j}(\infty)\right)^{2}\right]<\infty \text {. }
$$

From Eqs. 3.10, 3.11, 3.12 and 3.15 we get that

$$
\begin{aligned}
B= & \frac{1}{\epsilon^{2}} \sum_{i} \frac{1}{i^{4}}\left\{\sum _ { n = 1 } ^ { i ^ { 2 } } \sum _ { l = 1 } ^ { i ^ { 2 } } \left[-q_{j}(\infty) \mathbb{E}\left[q_{j}(0, n)\right]+q_{j}^{2}(\infty)-q_{j}(\infty) \mathbb{E}\left[q_{j}(0, l)\right]\right.\right. \\
& \left.\left.+\mathbb{E}\left[q_{j}^{(j)}(n \vee l, n \wedge l)\right] \mathbb{E}\left[q_{j}(0, n \vee l)\right]\right]\right\} \\
\leq & \frac{1}{\epsilon^{2}} \sum_{i} \frac{1}{i^{4}}\left\{\sum _ { n = 1 } ^ { i ^ { 2 } } \sum _ { l = 1 } ^ { i ^ { 2 } } \left\{\left|q_{j}(\infty)-\mathbb{E}\left[q_{j}(0, n)\right]\right|+\left|q_{j}(\infty)-\mathbb{E}\left[q_{j}(0, l)\right]\right|\right.\right. \\
& \left.\left.+\left|\mathbb{E}\left[q_{j}(0, n \vee l)\right]-q_{j}(\infty)\right|+\left|\mathbb{E}\left[q_{j}^{(j)}(n \vee l, n \wedge l)\right]-q_{j}(\infty)\right|\right\}\right\} .
\end{aligned}
$$


From Theorem 10 we get that there exists constants $c>0$ and $0<b<1$ such that

$$
\begin{aligned}
& \left|q_{j}(\infty)-\mathbb{E}\left[q_{j}(0, n)\right]\right| \leq c b^{n} ;\left|q_{j}(\infty)-\mathbb{E}\left[q_{j}(0, l)\right]\right| \leq c b^{l} \text { and } \\
& \left|\mathbb{E}\left[q_{j}(0, n \vee l)\right]-q_{j}(\infty)\right| \leq c b^{n \vee l} .
\end{aligned}
$$

From Theorem 9 we get that there exists constants $c_{1}>0$ and $0<b_{1}<1$ such that

$$
\left|\mathbb{E}\left[q_{j}^{(j)}(n \vee l, n \wedge l)\right]-q_{j}(\infty)\right| \leq c_{1} b_{1}^{n \wedge l-n \vee l} .
$$

From Eqs. 3.24, 3.25 and 3.26 we get that

$$
B \leq \frac{1}{\epsilon^{2}} \sum_{i} \frac{1}{i^{4}} \sum_{n=1}^{i^{2}} \sum_{l=1}^{i^{2}}\left[c b^{n}+c b^{l}+c b^{n \vee l}+c_{1} b_{1}^{n \wedge l-n \vee l}\right]<\infty .
$$

Hence, we have proved that

$$
\frac{1}{i^{2}} \sum_{s=1}^{i^{2}} u_{j}(s) \stackrel{a . s .}{\rightarrow} q_{j}(\infty)
$$

We have that

$$
\sum_{s=1}^{i^{2}} u_{j}(s) \text { is monotonic non-decreasing, }
$$

therefore

$$
\sum_{s=1}^{i^{2}} u_{j}(s) \leq \sum_{s=1}^{t} u_{j}(s) \leq \sum_{s=1}^{(i+1)^{2}} u_{j}(s) \text { if } i^{2} \leq t \leq(i+1)^{2},
$$

from which we get

$$
\frac{1}{(i+1)^{2}} \sum_{s=1}^{i^{2}} u_{j}(s) \leq \frac{1}{t} \sum_{s=1}^{t} u_{j}(s) \leq \frac{1}{i^{2}} \sum_{s=1}^{(i+1)^{2}} u_{j}(s) \text { if } i^{2} \leq t \leq(i+1)^{2} .
$$

In Eq. 3.30 let $t \rightarrow \infty$, use the fact that $\lim _{i \rightarrow \infty}\left(i^{2} /(i+1)^{2}\right) \rightarrow 1$, and relation (3.27) to get

$$
\frac{1}{t} \sum_{s=1}^{t} u_{j}(s) \stackrel{\text { a.s. }}{\rightarrow} q_{j}(\infty)
$$

\section{Convergence in the Cesaro Sense for Cyclic NHMS}

In the present section we study convergence of the relative population structure in the Cesaro sense for an NHMS which undergoes a cyclic behavior. This is a founding step in order to study Laws of Large Numbers in Cyc-NHMS in the next section. The importance of cyclic behavior was firstly stressed in Bartholomew (1982) p.71. The motive was Gani's (1963) study of student enrolment at Michigan state University. A general theorem for the limiting behavior of the expected population structure for a Cyc-NHMS was given in Vassiliou (1984). Also, the asymptotic variability of nonhomogeneous Markov systems under cyclic behavior was studied in Vassiliou (1986). Georgiou and Tsantas (1996) studied asymptotic attainability of nonstationary cyclic Markov systems as a natural extension of Cyc-NHMS.

We now provide the definition of a Cyc-NHMS 
Definition 4 Let a probability space $(\Omega, \mathcal{F}, \mathbb{P})$ and an NHMS as defined in Section 2. We say that the NHMS undergoes a cyclic behavior with period $d$ if and only if for all $m=1,2, \ldots$ and $s=0,1, \ldots, d-1$

$$
\mathbf{P}(m d+s)=\mathbf{P}(s) ; \mathbf{p}_{k+1}^{\top}(m d+s)=\mathbf{p}_{k+1}^{\top} ; \mathbf{p}_{0}(m d+s)=\mathbf{p}_{0} .
$$

It is apparent that for a Cyc-NHMS with period $d$ we have that for all $m=1,2, \ldots$ and $s=0,1, \ldots, d-1$

$$
\mathbf{Q}(m d+s)=\mathbf{Q}(s) .
$$

We now define the following stochastic matrices

$$
\begin{aligned}
\mathbf{Q}_{0} & =\mathbf{Q}(0) \mathbf{Q}(1) \ldots \mathbf{Q}(d-1) ; \mathbf{Q}_{1}=\mathbf{Q}(1) \mathbf{Q}(2) \ldots \mathbf{Q}(d-1) \mathbf{Q}(0), \ldots, \\
\mathbf{Q}_{d-1} & =\mathbf{Q}(d-1) \mathbf{Q}(0) \ldots \mathbf{Q}(d-2) .
\end{aligned}
$$

It is well known that if $\mathbf{Q}_{0}$ is a regular stochastic matrix then $\lim _{t \rightarrow \infty} \mathbf{Q}_{0}^{t}=\mathbf{Q}_{0}^{\infty}$ a stable matrix or equivalently

$$
\lim _{t \rightarrow \infty}\left\|\mathbf{Q}_{0}^{t}-\mathbf{Q}_{0}^{\infty}\right\|=0
$$

We will now provide the following Proposition:

Proposition 1 Let a probability space $(\Omega, \mathcal{F}, \mathbb{P})$ and a Cyc-NHMS. If $\mathbf{Q}_{0}$ is a regular stochastic matrix then

$$
\lim _{t \rightarrow \infty}\left\|\mathbf{Q}_{i}^{t}-\mathbf{Q}_{i}^{\infty}\right\|=0, \text { for } i=1,2, \ldots, d-1 .
$$

where

$$
\mathbf{Q}_{i}^{\infty}=\mathbf{Q}_{0}^{\infty}\left(\prod_{j=0}^{i-1} \mathbf{Q}(j)\right), \text { for } i=1,2, \ldots, d-1
$$

Proof Since $\mathbf{Q}_{0}^{\infty}$ is a stable stochastic matrix we have that

$$
\mathbf{Q}_{i}^{\infty}=\left(\prod_{j=i}^{d-1} \mathbf{Q}(j)\right) \mathbf{Q}_{0}^{\infty}\left(\prod_{j=0}^{i-1} \mathbf{Q}(j)\right),
$$

therefore we have that

$$
\begin{aligned}
& \mathbf{Q}_{i}^{t}=\underbrace{\mathbf{Q}_{i} \mathbf{Q}_{i} \ldots \mathbf{Q}_{i}}_{t \text {-times }}= \\
& =\mathbf{Q}(i) \mathbf{Q}(i+1) \ldots \mathbf{Q}(d-1) \mathbf{Q}(0) \mathbf{Q}(1) \ldots \mathbf{Q}(i-1) \\
& \times \mathbf{Q}(i) \mathbf{Q}(i+1) \ldots \mathbf{Q}(d-1) \mathbf{Q}(0) \mathbf{Q}(1) \ldots \mathbf{Q}(i-1) \\
& \times \\
& \times \mathbf{Q}(i) \mathbf{Q}(i+1) \ldots \mathbf{Q}(d-1) \mathbf{Q}(0) \mathbf{Q}(1) \ldots \mathbf{Q}(i-1) \\
& \times\left(\prod_{j=i}^{d-1} \mathbf{Q}(j)\right) \mathbf{Q}_{0}^{t-1}\left(\prod_{j=0}^{i-1} \mathbf{Q}(j)\right)
\end{aligned}
$$


hence, for every $\epsilon>0$ there is a $t_{0}$ such that for $t \geq t_{0}$

$$
\begin{aligned}
\left\|\mathbf{Q}_{i}^{t}-\mathbf{Q}_{i}^{\infty}\right\| & =\left\|\left(\prod_{j=i}^{d-1} \mathbf{Q}(j)\right) \mathbf{Q}_{0}^{t-1}\left(\prod_{j=0}^{i-1} \mathbf{Q}(j)\right)-\left(\prod_{j=i}^{d-1} \mathbf{Q}(j)\right) \mathbf{Q}_{0}^{\infty}\left(\prod_{j=0}^{i-1} \mathbf{Q}(j)\right)\right\| \\
& \leq\left\|\mathbf{Q}_{0}^{t}-\mathbf{Q}_{0}^{\infty}\right\|<\epsilon .
\end{aligned}
$$

From Vassiliou and Georgiou (1990) p.541 we get the following Lemma

Lemma 1 Let a probability space $(\Omega, \mathcal{F}, \mathbb{P})$ and a NHMS be defined in Section 2. Suppose that the sequence

$$
\left\{\frac{\Delta T(t)}{T(t)}\right\}_{t=0}^{\infty}
$$

converges to zero geometrically fast with $T(t) \geq T(t-1)$. Then $\{T(t)\}_{t=0}^{\infty}$ converges geometrically fast.

Remark 1 The assumption $\lim _{t \rightarrow \infty} \frac{\Delta T(t)}{T(t)}=0$ allows for $\lim _{t \rightarrow \infty} T(t)=\infty$.

We will now prove the following theorem

Theorem 13 Define a probability space $(\Omega, \mathcal{F}, \mathbb{P})$ and a Cyc-NHMS. If $(a) \mathbf{Q}_{0}=$ $\mathbf{Q}(0) \mathbf{Q}(1) \ldots \mathbf{Q}(d-1)$ is a regular stochastic matrix; $\lim _{t \rightarrow \infty} \frac{\Delta T(t)}{T(t)}=0$ with $\lim _{t \rightarrow \infty} T(t)=\infty$ then the sequence $\mathbb{E}[q(0, t)]$ splits into $d$ subsequences with limits

$$
\begin{aligned}
\mathbf{q}_{s}(\infty)= & \sum_{r=0}^{s-1} \mathbf{p}_{0}(r) s_{r} \mathbf{Q}_{r}^{(\infty)}\left(\prod_{j=r}^{s-1} \mathbf{Q}(j)\right) \\
& +\sum_{r=s}^{d-1} \mathbf{p}_{0}(r) s_{r} \mathbf{Q}_{r}^{(\infty)}\left(\prod_{j=r}^{d-1} \mathbf{Q}(j)\right)\left(\prod_{j=0}^{s-1} \mathbf{Q}(j)\right) \\
\text { for } s= & 0,1, \ldots, d-1 .
\end{aligned}
$$

(b) $\mathbf{Q}_{0}=\mathbf{Q}(0) \mathbf{Q}(1) \ldots \mathbf{Q}(d-1)$ is a regular stochastic matrix; $\lim _{t \rightarrow \infty} \frac{\Delta T(t)}{T(t)}=0$ geometrically fast then the sequence $\mathbb{E}[q(0, t)]$ splits into d subsequences with limits

$$
\begin{aligned}
\mathbf{q}_{s}(\infty)= & \frac{T(0)}{T} \mathbf{q}(0) \mathbf{Q}_{s-1}^{(\infty)}+\sum_{r=0}^{s-1} \mathbf{p}_{0}(r) s_{r} \mathbf{Q}_{r}^{(\infty)}\left(\prod_{j=r}^{s-1} \mathbf{Q}(j)\right) \\
& +\sum_{r=s}^{d-1} \mathbf{p}_{0}(r) s_{r} \mathbf{Q}_{r}^{(\infty)}\left(\prod_{j=r}^{d-1} \mathbf{Q}(j)\right)\left(\prod_{j=0}^{s-1} \mathbf{Q}(j)\right) \\
\text { for } s= & 0,1, \ldots, d-1 .
\end{aligned}
$$

Proof Without loss of generality assume that $t=m d+s$. Due to the fact that we have a Cyc-NHMS we get that

$$
\mathbf{Q}(0, m d+s-1)=\mathbf{Q}_{0}^{m}\left(\prod_{j=0}^{s-1} \mathbf{Q}(j)\right) .
$$


Since $\mathbf{Q}_{0}$ is a regular stochastic matrix it is easy using Eq. 4.6 to see that

$$
\lim _{m \rightarrow \infty}\left\|\mathbf{Q}(0, m d+s-1)-\mathbf{Q}_{s-1}^{\infty}\right\|=0 .
$$

Denote by

$$
\begin{aligned}
U(m d+s)= & \frac{1}{T(m d+s)} \sum_{\tau=1}^{m d+s} \Delta T(\tau-1) \mathbf{p}_{0}(\tau-1) \mathbf{Q}(\tau, m d+s-1) \\
= & \sum_{\tau=0}^{m-1} \sum_{r=0}^{d-1} \frac{\Delta T(\tau d+r)}{T(m d+s)} \mathbf{p}_{0}(\tau d+r) \mathbf{Q}(\tau d+r, m d+s-1) \\
& +\sum_{r=0}^{s-1} \frac{\Delta T(m d+r)}{T(m d+s)} \mathbf{p}_{0}(m d+r) \mathbf{Q}(m d+r, m d+s-1) .
\end{aligned}
$$

From Eq. 4.11 we get that

$$
\begin{aligned}
& \lim _{m \rightarrow \infty}\left\|\sum_{r=0}^{s-1} \frac{\Delta T(m d+r)}{T(m d+s)} \mathbf{p}_{0}(m d+r) \mathbf{Q}(m d+r, m d+s-1)\right\| \\
\leq & \lim _{m \rightarrow \infty} \sum_{r=0}^{s-1} \frac{\Delta T(m d+r)}{T(m d+s)}\left\|\mathbf{p}_{0}(m d+r)\right\|\|\mathbf{Q}(m d+r, m d+s-1)\| \\
\leq & \lim _{m \rightarrow \infty} \sum_{r=0}^{s-1} \frac{\Delta T(m d+r)}{T(m d+s)}=0 .
\end{aligned}
$$

Let $r \leq s-1$ then it is not difficult to see that

$$
\mathbf{Q}(\tau d+r, m d+s-1)=\mathbf{Q}_{r}^{(m-\tau)}\left(\prod_{j=r}^{s-1} \mathbf{Q}(j)\right) .
$$

On the other hand when $r>s-1$ then

$$
\mathbf{Q}(\tau d+r, m d+s-1)=\mathbf{Q}^{(m-\tau-1)}\left(\prod_{j=r}^{d-1} \mathbf{Q}(j)\right)\left(\prod_{j=0}^{s-1} \mathbf{Q}(j)\right)
$$

The expression in Eq. 4.10 could be written as

$$
\begin{aligned}
& \sum_{\tau=0}^{m-1} \sum_{r=0}^{d-1} \frac{\Delta T(\tau d+r)}{T(m d+s)} \mathbf{p}_{0}(\tau d+r) \mathbf{Q}(\tau d+r, m d+s-1) \\
= & \sum_{\tau=0}^{m-1} \sum_{r=0}^{s-1} \frac{\Delta T(\tau d+r)}{T(m d+s)} \mathbf{p}_{0}(\tau d+r) \mathbf{Q}(\tau d+r, m d+s-1) \\
& +\sum_{\tau=0}^{m-1} \sum_{r=s}^{d-1} \frac{\Delta T(\tau d+r)}{T(m d+s)} \mathbf{p}_{0}(\tau d+r) \mathbf{Q}(\tau d+r, m d+s-1) \\
= & U_{1}(m d+s)+U_{2}(m d+s) .
\end{aligned}
$$


We now have that

$$
\begin{aligned}
& \lim _{m \rightarrow \infty} \sum_{\tau=0}^{m-1} \sum_{r=0}^{s-1} \frac{\Delta T(\tau d+r)}{T(m d+s)} \mathbf{p}_{0}(\tau d+r) \mathbf{Q}(\tau d+r, m d+s-1) \\
& =(\text { from the fact that we have a Cyc-NHMS and (4.13)) } \\
& =\sum_{r=0}^{s-1} \mathbf{p}_{0}(r) \lim _{m \rightarrow \infty} \sum_{\tau=0}^{m-1} \frac{\Delta T(\tau d+r)}{T(m d+s)} \mathbf{Q}_{r}^{(m-\tau)}\left(\prod_{j=r}^{s-1} \mathbf{Q}(j)\right) .
\end{aligned}
$$

Now the series

$$
\sum_{\tau=0}^{m-1} \frac{\Delta T(\tau d+r)}{T(m d+s)} \text { for } r=0,1, \ldots, d-1,
$$

is bounded by the series

$$
\sum_{\tau=0}^{t} \frac{\Delta T(\tau)}{T(t)} \leq 1
$$

hence

$$
\lim _{m \rightarrow \infty} \sum_{\tau=0}^{m-1} \frac{\Delta T(\tau d+r)}{T(m d+s)} \leq 1
$$

since $\{T(t)\}_{t=0}^{\infty}$ is a monotonically increasing function of $t$, we have $\frac{\Delta T(\tau d+r)}{T(m d+s)} \geq 0$ and consequently the series in Eq. 4.18 is converging and denote by

$$
s_{r}=\lim _{m \rightarrow \infty} \sum_{\tau=0}^{m-1} \frac{\Delta T(\tau d+r)}{T(m d+s)} \text { for } r=0,1, \ldots, d-1 .
$$

We now have that

$$
\begin{aligned}
& \left\|\sum_{\tau=0}^{m-1} \frac{\Delta T(\tau d+r)}{T(m d+s)} \mathbf{Q}_{r}^{(m-\tau)}\left(\prod_{j=r}^{s-1} \mathbf{Q}(j)\right)-\sum_{\tau=0}^{m-1} \frac{\Delta T(\tau d+r)}{T(m d+s)} \mathbf{Q}_{r}^{(\infty)}\left(\prod_{j=r}^{s-1} \mathbf{Q}(j)\right)\right\| \\
& \leq \sum_{\tau=0}^{m-1} \frac{\Delta T(\tau d+r)}{T(m d+s)}\left\|\mathbf{Q}_{r}^{(m-\tau)}-\mathbf{Q}_{r}^{(\infty)}\right\| .
\end{aligned}
$$

From Proposition 1, Eqs. 4.16, 4.18 and 4.20 we get that

$$
\lim _{m \rightarrow \infty} U_{1}(m d+s)=\sum_{r=0}^{s-1} \mathbf{p}_{0}(r) s_{r} \mathbf{Q}_{r}^{(\infty)}\left(\prod_{j=r}^{s-1} \mathbf{Q}(j)\right) \quad \text { for } r \leq s-1 .
$$

In a similar way we arrive at

$$
\lim _{m \rightarrow \infty} U_{2}(m d+s)=\sum_{r=s}^{d-1} \mathbf{p}_{0}(r) s_{r} \mathbf{Q}_{r}^{(\infty)}\left(\prod_{j=r}^{d-1} \mathbf{Q}(j)\right)\left(\prod_{j=0}^{s-1} \mathbf{Q}(j)\right) .
$$


Hence, from Eqs. 4.10, 4.11 and 4.15 we get that

$$
\begin{aligned}
\lim _{m \rightarrow \infty} U(m d+s)= & \sum_{r=0}^{s-1} \mathbf{p}_{0}(r) s_{r} \mathbf{Q}_{r}^{(\infty)}\left(\prod_{j=r}^{s-1} \mathbf{Q}(j)\right) \\
& +\sum_{r=s}^{d-1} \mathbf{p}_{0}(r) s_{r} \mathbf{Q}_{r}^{(\infty)}\left(\prod_{j=r}^{d-1} \mathbf{Q}(j)\right)\left(\prod_{j=0}^{s-1} \mathbf{Q}(j)\right) .
\end{aligned}
$$

Now from Eqs. 2.6 and 4.23 we get that for $\lim _{t \rightarrow \infty} \frac{\Delta T(t)}{T(t)}=0$ and $\lim _{t \rightarrow \infty} T(t)=\infty$ the sequence $\mathbb{E}[q(0, t)]$ splits into $d$ subsequences with limits

$$
\begin{gathered}
\mathbf{q}_{s}(\infty)=\sum_{r=0}^{s-1} \mathbf{p}_{0}(r) s_{r} \mathbf{Q}_{r}^{(\infty)}\left(\prod_{j=r}^{s-1} \mathbf{Q}(j)\right) \\
+\sum_{r=s}^{d-1} \mathbf{p}_{0}(r) s_{r} \mathbf{Q}_{r}^{(\infty)}\left(\prod_{j=r}^{d-1} \mathbf{Q}(j)\right)\left(\prod_{j=0}^{s-1} \mathbf{Q}(j)\right) . \\
\text { for } s=0,1, \ldots, d-1 .
\end{gathered}
$$

If $\lim _{t \rightarrow \infty} \frac{\Delta T(t)}{T(t)}=0$ in a geometrical rate then by Lemma $1 \lim _{t \rightarrow \infty} T(t)=T$ and the sequence $\mathbb{E}[q(0, t)]$ splits into $d$ subsequences with limits

$$
\begin{gathered}
\mathbf{q}_{s}(\infty)=\frac{T(0)}{T} \mathbf{q}(0) \mathbf{Q}_{s-1}^{(\infty)}+\sum_{r=0}^{s-1} \mathbf{p}_{0}(r) s_{r} \mathbf{Q}_{r}^{(\infty)}\left(\prod_{j=r}^{s-1} \mathbf{Q}(j)\right) \\
+\sum_{r=s}^{d-1} \mathbf{p}_{0}(r) s_{r} \mathbf{Q}_{r}^{(\infty)}\left(\prod_{j=r}^{d-1} \mathbf{Q}(j)\right)\left(\prod_{j=0}^{s-1} \mathbf{Q}(j)\right) \\
\text { for } s=0,1, \ldots, d-1 .
\end{gathered}
$$

We will now introduce the concept of Cesaro strongly ergodic for a Cycl-NHMS:

Definition 5 A Cycl-NHMS is called Cesaro strongly ergodic if there exists a vector $\mathbf{q}(\infty)$ such that

$$
\lim _{t \rightarrow \infty}\left\|\frac{1}{t} \sum_{n=0}^{t} \mathbb{E}[\mathbf{q}(0, n)]-\mathbf{q}(\infty)\right\|=0 .
$$

We call the $\mathbf{q}(\infty)$ the Cyclic strong run distribution for the NHMS.

We will now provide a basic theorem on the Cesaro convergence for a Cycl-NHMS.

Theorem 14 Consider a Cycl-NHMS and let that:

(a) $\mathbf{Q}_{0}=\mathbf{Q}(0) \mathbf{Q}(1) \ldots \mathbf{Q}(d-1)$ is a regular stochastic matrix;

(b) $\lim _{t \rightarrow \infty} \frac{\Delta T(t)}{T(t)}=0$ 
then the Cycl-NHMS is Cesaro strongly ergodic in the sense that

$$
\lim _{t \rightarrow \infty}\left\|\frac{1}{t} \sum_{n=0}^{t} \mathbb{E}[\mathbf{q}(0, n)]-\frac{1}{d} \sum_{s=0}^{d-1} \mathbf{q}_{s}(\infty)\right\|=0,
$$

where

$$
\begin{aligned}
\mathbf{q}_{s}(\infty)= & \sum_{r=0}^{s-1} \mathbf{p}_{0}(r) s_{r} \mathbf{Q}_{r}^{(\infty)}\left(\prod_{j=r}^{s-1} \mathbf{Q}(j)\right) \\
& +\sum_{r=s}^{d-1} \mathbf{p}_{0}(r) s_{r} \mathbf{Q}_{r}^{(\infty)}\left(\prod_{j=r}^{d-1} \mathbf{Q}(j)\right)\left(\prod_{j=0}^{s-1} \mathbf{Q}(j)\right) .
\end{aligned}
$$

If (a) $\mathbf{Q}_{0}=\mathbf{Q}(0) \mathbf{Q}(1) \ldots \mathbf{Q}(d-1)$ is a regular stochastic matrix; $(\hat{b}) \lim _{t \rightarrow \infty} \frac{\Delta T(t)}{T(t)}=0$ geometrically fast then the Cycl-NHMS is Cesaro strongly ergodic in the sense that

$$
\lim _{t \rightarrow \infty}\left\|\frac{1}{t} \sum_{n=0}^{t} \mathbb{E}[\mathbf{q}(0, n)]-\frac{1}{d} \sum_{s=0}^{d-1} \mathbf{q}_{s}(\infty)\right\|=0
$$

where

$$
\begin{aligned}
\mathbf{q}_{s}(\infty)= & \frac{T(0)}{T} \mathbf{q}(0) \mathbf{Q}_{s-1}^{(\infty)}+\sum_{r=0}^{s-1} \mathbf{p}_{0}(r) s_{r} \mathbf{Q}_{r}^{(\infty)}\left(\prod_{j=r}^{s-1} \mathbf{Q}(j)\right) \\
& +\sum_{r=s}^{d-1} \mathbf{p}_{0}(r) s_{r} \mathbf{Q}_{r}^{(\infty)}\left(\prod_{j=r}^{d-1} \mathbf{Q}(j)\right)\left(\prod_{j=0}^{s-1} \mathbf{Q}(j)\right)
\end{aligned}
$$

Proof We start with the first part, that is $(a)$ and $(b)$ hold. Since the Cycl-NHMS is of finite size it is sufficient to show that

$$
\lim _{t \rightarrow \infty} \frac{1}{t} \sum_{n=0}^{t} \mathbb{E}[\mathbf{q}(0, n)]=\frac{1}{d} \sum_{s=0}^{d-1} \mathbf{q}_{s}(\infty) .
$$

Let $\left[\frac{a}{b}\right]$ the integer part of the division then we have that

$$
\frac{1}{t} \sum_{n=0}^{t} \mathbb{E}[\mathbf{q}(0, n)]=\frac{1}{t} \sum_{n=0}^{d[t / d]-1} \mathbb{E}[\mathbf{q}(0, n)]+\frac{1}{t} \sum_{n=d[t / d]}^{t} \mathbb{E}[\mathbf{q}(0, n)] .
$$

Now we have that

$$
\begin{aligned}
\lim _{t \rightarrow \infty} \frac{1}{t} \sum_{n=0}^{d[t / d]-1} \mathbb{E}[\mathbf{q}(0, n)] & =\lim _{t \rightarrow \infty} \frac{1}{t} \sum_{s=0}^{d-1} \sum_{n=0}^{[t / d]-1} \mathbb{E}[\mathbf{q}(0, n d+s)] \\
& =\sum_{s=0}^{d-1} \lim _{t \rightarrow \infty} \frac{[t / d]}{t} \frac{1}{[t / d]} \sum_{n=0}^{[t / d]-1} \mathbb{E}[\mathbf{q}(0, n d+s)]
\end{aligned}
$$


From $(a),(b)$, Theorem 13 and the fact that the series is an arithmetic mean we get that

$$
\begin{aligned}
\lim _{t \rightarrow \infty} \frac{1}{[t / d]} \sum_{n=0}^{[t / d]-1} \mathbb{E}[\mathbf{q}(0, n d+s)]= & \sum_{r=0}^{s-1} \mathbf{p}_{0}(r) s_{r} \mathbf{Q}_{r}^{(\infty)}\left(\prod_{j=r}^{s-1} \mathbf{Q}(j)\right) \\
& +\sum_{r=s}^{d-1} \mathbf{p}_{0}(r) s_{r} \mathbf{Q}_{r}^{(\infty)}\left(\prod_{j=r}^{d-1} \mathbf{Q}(j)\right)\left(\prod_{j=0}^{s-1} \mathbf{Q}(j)\right) .
\end{aligned}
$$

Also $\lim _{t \rightarrow \infty}[t / d] / t=1 / d$ therefore from Eqs. 4.28 and 4.29 we get that

$$
\begin{aligned}
\lim _{t \rightarrow \infty} \frac{1}{t} \sum_{n=0}^{d[t / d]-1} \mathbb{E}[\mathbf{q}(0, n)]= & \frac{1}{d} \sum_{s=0}^{d-1} \sum_{r=0}^{s-1} \mathbf{p}_{0}(r) s_{r} \mathbf{Q}_{r}^{(\infty)}\left(\prod_{j=r}^{s-1} \mathbf{Q}(j)\right) \\
& +\frac{1}{d} \sum_{s=0}^{d-1} \sum_{r=s}^{d-1} \mathbf{p}_{0}(r) s_{r} \mathbf{Q}_{r}^{(\infty)}\left(\prod_{j=r}^{d-1} \mathbf{Q}(j)\right)\left(\prod_{j=0}^{s-1} \mathbf{Q}(j)\right) .
\end{aligned}
$$

Now it is easy to see that

$$
\lim _{t \rightarrow \infty} \frac{1}{t} \sum_{n=d[t / d]}^{t} \mathbb{E}[\mathbf{q}(0, n)]=0 .
$$

The second part of the Theorem is proved in a similar way.

\section{Laws of Large Numbers for a Cycl-NHMS}

We are now in a position to study the first Law of Large Numbers for a Cycl-NHMS. We will start with the mode of mean square convergence. Let $X_{t}, u_{j}(t), y_{j}(t)$ and $v_{j}(t)$ be defined as in Section 3. We will now provide and prove the following theorem of the Law of Large Numbers

Theorem 15 Define a probability space $(\Omega, \mathcal{F}, \mathbb{P})$ and a Cyc-NHMS. If $(a) \mathbf{Q}_{0}=$ $\mathbf{Q}(0) \mathbf{Q}(1) \ldots \mathbf{Q}(d-1)$ is a regular stochastic matrix and $\lim _{t \rightarrow \infty} \frac{\Delta T(t)}{T(t)}=0$ then

$$
v(t) \stackrel{L^{2}}{\rightarrow} \frac{1}{d} \sum_{s=0}^{d-1} \mathbf{q}_{s}(\infty)
$$

where $\mathbf{q}_{s}(\infty)$ is given by Eq. 4.24 if in addition $T(t) \rightarrow_{t \rightarrow \infty} \infty$; and where $\mathbf{q}_{s}(\infty)$ is given by Eq. 4.25 if $\lim _{t \rightarrow \infty} \frac{\Delta T(t)}{T(t)}=0$ geometrically fast.

Proof It is equivalent to show that

$$
\lim _{t \rightarrow \infty} \mathbb{E}\left[\left(v(t)-\frac{1}{d} \sum_{s=0}^{d-1} \mathbf{q}_{s}(\infty)\right)^{2}\right]=0 .
$$

Since the dimensions of the vectors are finite it is equivalent to show that

$$
\lim _{t \rightarrow \infty} \mathbb{E}\left[\left(v_{j}(t)-\frac{1}{d} \sum_{s=0}^{d-1} q_{s j}(\infty)\right)^{2}\right]=0 \text { for } j=1,2, \ldots, k .
$$


We have that

$$
\begin{aligned}
\mathbb{E}\left[\left(v_{j}(t)-\frac{1}{d} \sum_{s=0}^{d-1} q_{s j}(\infty)\right)^{2}\right]= & \mathbb{E}\left[\left(\sum_{n=1}^{t} \frac{u_{j}(n)}{t}-\frac{1}{d} \sum_{s=0}^{d-1} q_{s j}(\infty)\right)^{2}\right] \\
= & \frac{1}{t^{2}} \mathbb{E}\left[\left(\sum_{n=1}^{t}\left(u_{j}(n)-\frac{1}{d} \sum_{s=0}^{d-1} q_{s j}(\infty)\right)\right)^{2}\right] \\
= & \frac{1}{t^{2}} \mathbb{E}\left[\left(\sum_{n=1}^{t}\left(u_{j}(n)-\frac{1}{d} \sum_{s=0}^{d-1} q_{s j}(\infty)\right)\right)\right. \\
& \left.\times\left(\sum_{l=1}^{t}\left(u_{j}(l)-\frac{1}{d} \sum_{s=0}^{d-1} q_{s j}(\infty)\right)\right)\right]
\end{aligned}
$$

Therefore we get that

$$
\begin{aligned}
\mathbb{E}\left[\left(v_{j}(t)-\frac{1}{d} \sum_{s=0}^{d-1} q_{s j}(\infty)\right)^{2}\right]= & \frac{1}{t^{2}} \sum_{n=1}^{t} \sum_{l=1}^{t} \mathbb{E}\left[u_{j}(n) u_{j}(l)\right] \\
& -\frac{1}{t^{2} d} \sum_{s=0}^{d-1} \sum_{n=1}^{t} \sum_{l=1}^{t} q_{s j}(\infty) \mathbb{E}\left[u_{j}(n)\right] \\
& -\frac{1}{t^{2} d} \sum_{s=0}^{d-1} \sum_{n=1}^{t} \sum_{l=1}^{t} q_{s j}(\infty) \mathbb{E}\left[u_{j}(l)\right] \\
& +\frac{1}{t^{2}} \sum_{n=1}^{t} \sum_{l=1}^{t}\left(\frac{1}{d} \sum_{s=0}^{d-1} q_{s j}(\infty)\right)^{2} .
\end{aligned}
$$

We start with relation (5.5)

$$
\begin{aligned}
\lim _{t \rightarrow \infty}\left\{-\frac{1}{t^{2} d} \sum_{s=0}^{d-1} \sum_{n=1}^{t} \sum_{l=1}^{t} q_{s j}(\infty) \mathbb{E}\left[u_{j}(n)\right]\right\} & =\lim _{t \rightarrow \infty}\left\{-\frac{1}{d} \sum_{s=0}^{d-1} q_{s j}(\infty) \frac{1}{t} \sum_{n=1}^{t} \mathbb{E}\left[u_{j}(n)\right]\right\} \\
& =\lim _{t \rightarrow \infty}\left\{-\frac{1}{d} \sum_{s=0}^{d-1} q_{s j}(\infty) \frac{1}{t} \sum_{n=1}^{t} \mathbb{E}\left[\mathbb{E}\left[u_{j}(n) \mid \mathbf{q}(0)\right]\right]\right\} \\
& =\lim _{t \rightarrow \infty}\left\{-\frac{1}{d} \sum_{s=0}^{d-1} q_{s j}(\infty) \frac{1}{t} \sum_{n=1}^{t} \mathbb{E}\left[\mathbb{P}\left(X_{n}=j\right) \mid \mathbf{q}(0)\right]\right\} \\
& =\lim _{t \rightarrow \infty}\left\{-\frac{1}{d} \sum_{s=0}^{d-1} q_{s j}(\infty) \frac{1}{t} \sum_{n=1}^{t} \mathbb{E}\left[q_{j}(0, n)\right]\right\} \\
& =\left(\text { by Theorem 14) }=-\left(\frac{1}{d} \sum_{s=0}^{d-1} q_{s j}(\infty)\right)^{2} .\right.
\end{aligned}
$$


Similarly we get that

$$
-\frac{1}{t^{2} d} \sum_{s=0}^{d-1} \sum_{n=1}^{t} \sum_{l=1}^{t} q_{s j}(\infty) \mathbb{E}\left[u_{j}(l)\right]=-\left(\frac{1}{d} \sum_{s=0}^{d-1} q_{s j}(\infty)\right)^{2} .
$$

It is easy to see that

$$
\frac{1}{t^{2}} \sum_{n=1}^{t} \sum_{l=1}^{t}\left(\frac{1}{d} \sum_{s=0}^{d-1} q_{s j}(\infty)\right)^{2}=\left(\frac{1}{d} \sum_{s=0}^{d-1} q_{s j}(\infty)\right)^{2} .
$$

The term (5.4) could be written as

$$
\begin{aligned}
\lim _{t \rightarrow \infty}\left\{\frac{1}{t^{2}} \sum_{n=1}^{t} \sum_{l=1}^{t} \mathbb{E}\left[u_{j}(n) u_{j}(l)\right]\right\} & =\lim _{t \rightarrow \infty}\left\{\frac{1}{t^{2}} \sum_{n=1}^{t} \sum_{l=1}^{t} \mathbb{E}\left[\mathbb{E}\left[u_{j}(n) u_{j}(l) \mid \mathbf{q}(0)\right]\right]\right\} \\
& =B .
\end{aligned}
$$

Now from Eqs. 5.11 and 3.14 we get that

$$
\begin{aligned}
B & =\lim _{t \rightarrow \infty}\left\{\frac{1}{t^{2}} \sum_{n=1}^{t} \sum_{l=1}^{t} \mathbb{E}\left[\mathbb{P}\left[u_{j}(n) u_{j}(l) \mid \mathbf{q}(0)\right]\right]\right\} \\
& =(\text { from }(3.14)) \\
& =\lim _{t \rightarrow \infty}\left\{\frac{1}{t^{2}} \sum_{n=1}^{t} \sum_{l=1}^{t} \mathbb{E}\left[\mathbb{P}\left(X_{n \wedge l}=j \mid q_{j}(0, n \vee l)=1\right)\right] \mathbb{E}\left[\mathbb{P}\left[X_{n \vee l}=j \mid \mathbf{q}(0)\right]\right]\right\} \\
& =\lim _{t \rightarrow \infty}\left\{\frac{1}{t^{2}} \sum_{n=1}^{t} \sum_{l=1}^{t} \mathbb{E}\left[q_{j}^{(j)}(n \vee l, n \wedge l)\right] \mathbb{E}\left[q_{j}(0, n \vee l)\right]\right\} \\
& =\left(\operatorname{since} q_{j}^{(j)}(s, t)=0 \text { for } s>t\right) \\
& =\lim _{t \rightarrow \infty}\left\{\frac{1}{t^{2}} \sum_{n=1}^{t} \sum_{l=n}^{t} \mathbb{E}\left[q_{j}^{(j)}(n, l)\right] \mathbb{E}\left[q_{j}(0, n)\right]\right\} \\
& =\lim _{t \rightarrow \infty}\left\{\frac{1}{t} \sum_{n=1}^{t} \mathbb{E}\left[q_{j}(0, n)\right] \frac{1}{t} \sum_{l=n}^{t} \mathbb{E}\left[q_{j}^{(j)}(n, l)\right]\right\} \\
& =(\text { by Theorem } 14 \text { and } 13) \\
& =\left(\frac{1}{d} \sum_{s=0}^{d-1} q_{s j}(\infty)\right)\left(\frac{1}{d} \sum_{s=0}^{d-1} q_{s j}(\infty)\right) \\
& =\left(\frac{1}{d} \sum_{s=0}^{d-1} q_{s j}(\infty)\right) .
\end{aligned}
$$

From Eqs. 5.3, 5.4,.., 5.12 we get Eq. 5.1 and that completes the proof.

We will now establish under what conditions the $L^{2}$ convergence of the Law of Large numbers we proved in Theorem 15 holds for almost sure convergence also. In order to do 
so we need the following founding Theorem which provides the conditions under which the Cesaro convergence in Theorem 14 is with geometrical rate.

Theorem 16 Define a probability space $(\Omega, \mathcal{F}, \mathbb{P})$ and a Cyc-NHMS. If $(a) \mathbf{Q}_{0}=$ $\mathbf{Q}(0) \mathbf{Q}(1) \ldots \mathbf{Q}(d-1)$ is a regular stochastic matrix and $\lim _{t \rightarrow \infty} \frac{\Delta T(t)}{T(t)}=0$ geometrically fast then

$$
\lim _{t \rightarrow \infty}\left\|\frac{1}{t} \sum_{n=0}^{t} \mathbb{E}[\mathbf{q}(0, n)]-\frac{1}{d} \sum_{s=0}^{d-1} \mathbf{q}_{s}(\infty)\right\|=0,
$$

in a geometrical rate.

Proof From the fact that $\mathbf{Q}_{0}$ is a regular stochastic matrix we know that

$$
\text { There exists } c_{0}>0 \text { and } 0<b_{0}<1 \text { such that }\left\|\mathbf{Q}_{0}^{t}-\mathbf{Q}_{0}^{(\infty)}\right\| \leq c_{0} b_{0}^{t} \text {. }
$$

From the end of the proof of Proposition 1 we get that

$$
\left\|\mathbf{Q}_{i}^{t}-\mathbf{Q}_{i}^{(\infty)}\right\| \leq c_{0} b_{0}^{t-1}
$$

hence the convergence is geometric. Now we have

$$
\begin{aligned}
& \left\|\mathbf{Q}(0, m d+s-1)-\mathbf{Q}_{0}^{(\infty)}\left(\prod_{j=0}^{s-1} \mathbf{Q}(j)\right)\right\| \\
& =\left\|\mathbf{Q}_{0}^{m}\left(\prod_{j=0}^{s-1} \mathbf{Q}(j)\right)-\mathbf{Q}_{0}^{(\infty)}\left(\prod_{j=0}^{s-1} \mathbf{Q}(j)\right)\right\| \leq\left\|\mathbf{Q}_{0}^{m}-\mathbf{Q}_{0}^{(\infty)}\right\| \leq c_{0} b_{0}^{m} .
\end{aligned}
$$

From Vassiliou and Georgiou (1990) we know that since $\lim _{t \rightarrow \infty} \Delta T(t) / T(t)=0$ geometrically fast, then the sequence $\{T(t)\}_{t=0}^{\infty}$ converges geometrically fast to a positive scalar $T$ and so there exists $c_{1}>0$ and $0<b_{1}<1$ such that

$$
\left|\frac{1}{T(t)}-\frac{1}{T}\right|<c_{1} b_{1}^{t} .
$$

We start with the first part of the right hand side of Eq. 2.6 for the case of Cycl-NHMS:

$$
\begin{aligned}
& \left\|\frac{T(0)}{T(m d+s)} \mathbf{q}(0) \mathbf{Q}(0, m d+s-1)-\frac{T(0)}{T} \mathbf{q}(0) \mathbf{Q}_{s-1}^{(\infty)}\right\| \\
& \leq T(0)\|\mathbf{q}(0)\|\left\|\frac{1}{T(m d+s)} \mathbf{Q}(0, m d+s-1)-\frac{1}{T} \mathbf{Q}_{s-1}^{(\infty)}\right\| \\
& \leq\left|\frac{1}{T(m d+s)}-\frac{1}{T}\right|\|\mathbf{Q}(0, m d+s-1)\|+\left|\frac{1}{T}\right|\left\|\mathbf{Q}(0, m d+s-1)-\mathbf{Q}_{s-1}^{(\infty)}\right\| \\
& =(\text { from (5.15) and }(5.16)) \leq c_{2} b_{2} \text { with } c_{2}>0 \text { and } 0<b_{2}<1 .
\end{aligned}
$$


Now we have that

$$
\begin{aligned}
& \| \frac{1}{T(m s+s)} \sum_{\tau=0}^{m-1} \sum_{r=0}^{s-1} \Delta T(\tau d+r) \mathbf{p}_{0}(\tau d+r) \mathbf{Q}(\tau d+r, m d+s-1) \\
& -\frac{1}{T} \sum_{\tau=0}^{m-1} \sum_{r=0}^{s-1} \Delta T(\tau d+r) \mathbf{p}_{0}(r) \mathbf{Q}_{r}^{(\infty)}\left(\prod_{j=r}^{s-1} \mathbf{Q}(j)\right) \| \\
& \leq \| \frac{1}{T(m s+s)} \sum_{\tau=0}^{m-1} \sum_{r=0}^{s-1} \Delta T(\tau d+r) \mathbf{p}_{0}(\tau d+r) \mathbf{Q}(\tau d+r, m d+s-1) \\
& -\frac{1}{T} \sum_{\tau=0}^{m-1} \sum_{r=0}^{s-1} \Delta T(\tau d+r) \mathbf{p}_{0}(\tau d+r) \mathbf{Q}(\tau d+r, m d+s-1) \| \\
& +\| \frac{1}{T} \sum_{\tau=0}^{m-1} \sum_{r=0}^{s-1} \Delta T(\tau d+r) \mathbf{p}_{0}(\tau d+r) \mathbf{Q}(\tau d+r, m d+s-1) \\
& -\frac{1}{T} \sum_{\tau=0}^{m-1} \sum_{r=0}^{s-1} \Delta T(\tau d+r) \mathbf{p}_{0}(r) \mathbf{Q}_{r}^{(\infty)}\left(\prod_{j=r}^{s-1} \mathbf{Q}(j)\right) \|=I_{1}+I_{2}
\end{aligned}
$$

From relation (5.19) we get that

$$
\begin{aligned}
I_{1} & \leq\left|\frac{1}{T(m d+s)}-\frac{1}{T}\right| \sum_{\tau=0}^{m-1} \sum_{r=0}^{s-1}\left\|\Delta T(\tau d+r) \mathbf{p}_{0}(\tau d+r) \mathbf{Q}(\tau d+r, m d+s-1)\right\| \\
& \leq\left|\frac{1}{T(m d+s)}-\frac{1}{T}\right| \sum_{\tau=0}^{m-1} \sum_{r=0}^{s-1} \Delta T(\tau d+r)\left\|\mathbf{p}_{0}(\tau d+r)\right\|\|\mathbf{Q}(\tau d+r, m d+s-1)\| \\
& \leq\left|\frac{1}{T(m d+s)}-\frac{1}{T}\right|[T-T(0)] \leq c_{2} b_{2}^{m d+s} .
\end{aligned}
$$

Also

$$
\begin{aligned}
I_{2} & \leq \frac{1}{T} \sum_{\tau=0}^{m-1} \sum_{r=0}^{s-1} \Delta T(\tau d+r)\left\|\mathbf{p}_{0}(r)\right\|\left\|\mathbf{Q}(\tau d+r, m d+s-1)-\mathbf{Q}_{r}^{(\infty)}\left(\prod_{j=r}^{s-1} \mathbf{Q}(j)\right)\right\| \\
& \leq \frac{1}{T} \sum_{\tau=0}^{m-1} \sum_{r=0}^{s-1} \Delta T(\tau d+r)\left\|\mathbf{Q}_{r}^{(m-\tau)}-\mathbf{Q}_{r}^{(\infty)}\right\| .
\end{aligned}
$$

Assume that for $m \geq t_{0}:\left\|\mathbf{Q}_{r}^{(m-\tau)}-\mathbf{Q}_{r}^{(\infty)}\right\| \leq c_{3} b_{3}^{m-t_{0}}$ then

$$
\begin{aligned}
I_{2} \leq & \frac{1}{T} \sum_{\tau=0}^{m-t_{0}} \sum_{r=0}^{s-1} \Delta T(\tau d+r)\left\|\mathbf{Q}_{r}^{(m-\tau)}-\mathbf{Q}_{r}^{(\infty)}\right\| \\
& +\frac{1}{T} \sum_{\tau=m-t_{0}+1}^{m-1} \sum_{r=0}^{s-1} \Delta T(\tau d+r)\left\|\mathbf{Q}_{r}^{(m-\tau)}-\mathbf{Q}_{r}^{(\infty)}\right\|,
\end{aligned}
$$


from which we get that $I_{2}$ goes geometrically fast to zero. Therefore relation (5.18) converges geometrically fast to zero. In a similar way one could prove, that the convergence in (4.22) is geometrically fast. Hence, we get that

$$
\mathbb{E}[0, \mathbf{q}(m d+s)] \rightarrow_{m \rightarrow \infty} \mathbf{q}_{s}(\infty) \text { geometrically fast. }
$$

Now following the steps of Theorem 14 it is easy to show that

$$
\lim _{t \rightarrow \infty}\left\|\frac{1}{t} \sum_{n=0}^{t} \mathbb{E}[\mathbf{q}(0, n)]-\frac{1}{d} \sum_{s=0}^{d-1} \mathbf{q}_{s}(\infty)\right\|=0,
$$

in a geometrical rate.

Having proved this basic result, we are now in a position following the steps of the proof of Theorem 12 where the role of Theorems 9 and 10 is now played by Theorem16 to arrive at the following theorem.

Theorem 17 Define a probability space $(\Omega, \mathcal{F}, \mathbb{P})$ and a Cyc-NHMS. If $(a) \mathbf{Q}_{0}=$ $\mathbf{Q}(0) \mathbf{Q}(1) \ldots \mathbf{Q}(d-1)$ is a regular stochastic matrix and $\lim _{t \rightarrow \infty} \frac{\Delta T(t)}{T(t)}=0$ geometrically fast then

$$
v(t) \stackrel{\text { a.s }}{\rightarrow} \frac{1}{d} \sum_{s=0}^{d-1} \mathbf{q}_{s}(\infty) \quad \text { as } t \rightarrow \infty .
$$

\section{Applications}

\subsection{Geriatric and Stroke Patients}

In the present section we present two types of applications. The first one in the present subsection is a general Coxian phase type model, special forms of which has been used as stochastic models for geriatric patients and stroke patients by McClean and her co-authors McClean et al. (1998a, b), Taylor et al. (2000), Marshall et al. (2002), Marshall and McClean (2003, 2004), Garg et al. (2010), McClean et al. (2014a, b). In these applications in the basic model, we distinguish three states which are called hospital pathways. In the case of geriatric patients the states are the "Acute Care", the "Rehabilative" and the "Long Stay". From each state we have movements outside the hospital due to discharge or death. Also, geriatric patients may be thought of as progressing through stages of acute care, rehabilitation and long-stay care, where most patients are eventually rehabilitated and discharged. Geriatric medical services are an important asset in the care of the elderly, while at the same time they can be easy victims of the political pressure on savings in health care expenditure. Note that the number of pathways could be increased and the criterion is what best fits the data. However, there is no reason to consider a larger number of states in here due to the restriction of space. It is of importance in the best management of hospital resources and certainly to the benefit of geriatric patients to know the tendencies of the system in the long run. That is, what proportion of the total population is going to be in each state. In the case of stroke patients there are more types of transitions due to the nature of stroke, which allows for relapses and hence more transitions among the hospital pathways. The model we will illustrate in what follows could be easily adjusted for both cases. 
Consider a hospital which starts with $T(0)=400$ patients and in a very short time reaches its full capacity of 435 patients. That is $T(1)=420, T(2)=430, T(3)=435$. Assume three hospital pathways and let that the initial relative population structure is

$$
\mathbf{q}(0)=\left(\begin{array}{lll}
0.5 & 0.25 & 0.25
\end{array}\right) .
$$

The vast majority of new patients enter the system in hospital pathway one, either by taking an empty place or as a virtual replacement of a discharged patient, that is $q_{11}(t)=p_{11}(t)+$ $p_{14}(t) p_{01}(t)$. In here state 4 expresses the external environment. The entrance probabilities are

$$
\begin{aligned}
& \mathbf{p}_{0}(0)=\left(\begin{array}{llll}
0.6 & 0.3 & 0.1
\end{array}\right) ; \mathbf{p}_{0}(1)=\left(\begin{array}{lll}
0.5 & 0.3 & 0.2
\end{array}\right) ; \\
& \mathbf{p}_{0}(2)=\left(\begin{array}{lll}
0.75 & 0.25 & 0.1
\end{array}\right) \text { and } \mathbf{p}_{0}=\mathbf{p}_{0}(t)=\left(\begin{array}{lll}
0.7 & 0.2 & 0.1
\end{array}\right) \text {; }
\end{aligned}
$$

for $t=3,4, \ldots$. The form of the transition probability matrices according to the stochastic model for movements in the hospital is the following

$$
\mathbf{P}(t)=\left(\begin{array}{ccc}
p_{11}(t) & p_{12}(t) & p_{13}(t) \\
0 & p_{22}(t) & p_{23}(t) \\
0 & 0 & p_{33}(t)
\end{array}\right),
$$

also the inherent non-homogeneous Markov chain will evolve with the sequence of stochastic matrices $\mathbf{Q}(t)=\left\{q_{i j}(t)\right\}_{i, j \in S}$ where $q_{i j}(t)=p_{i j}(t)+p_{i 4}(t) p_{0 j}(t)$. We get the following typical set of $\mathbf{Q}(t)$ 's which are easily estimated from the data by its maximum likelihood estimates:

$$
\begin{gathered}
\mathbf{Q}(0)=\left(\begin{array}{lll}
0.7 & 0.2 & 0.1 \\
0.2 & 0.6 & 0.2 \\
0.6 & 0.1 & 0.3
\end{array}\right) ; \quad \mathbf{Q}(1)=\left(\begin{array}{ccc}
0.65 & 0.25 & 0.1 \\
0.1 & 0.7 & 0.2 \\
0.5 & 0.1 & 0.4
\end{array}\right) ; \\
\mathbf{Q}(2)=\left(\begin{array}{lll}
0.5 & 0.3 & 0.2 \\
0.2 & 0.5 & 0.3 \\
0.4 & 0.2 & 0.4
\end{array}\right) ; \quad \mathbf{Q}(t)=\mathbf{Q}=\left(\begin{array}{ccc}
0.6 & 0.3 & 0.1 \\
0.14 & 0.6 & 0.26 \\
0.49 & 0.14 & 0.37
\end{array}\right),
\end{gathered}
$$

for $t=2,3, \ldots$. The row of the stable matrix $\lim _{t \rightarrow \infty} \mathbf{Q}^{t}=\mathbf{Q}(\infty)=(\mathbf{q}(\infty) \mathbf{q}(\infty)$ $\mathbf{q}(\infty))^{\top}$ is

$$
\mathbf{q}(\infty)=\left(\begin{array}{lll}
0.41 & 0.37 & 0.22
\end{array}\right),
$$

where the convergence is geometrically fast, that is for $t=5$ it already converges. Now simulating Theorem 11 we find that

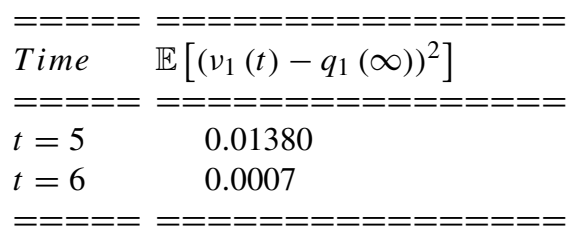

From the above table it is apparent that

$$
v_{1}(t) \stackrel{L^{2}}{\rightarrow} 0.41
$$

Analogous results are found also for the remaining of the hospital pathways. That is

$$
v(t) \stackrel{L^{2}}{\rightarrow}\left(\begin{array}{lll}
0.41 & 0.37 & 0.22
\end{array}\right) .
$$


Now, since as we have seen $\Delta T(t) / T(t) \rightarrow 0$ geometrically fast, that is, for $t=3$, and since as we have seen $\lim _{t \rightarrow \infty} \mathbf{Q}^{t}$ converges to $\mathbf{Q}(\infty)$ geometrically fast, that is, for $t=5$, then according to Theorem 12 we have

$$
v(t) \stackrel{a . s}{\rightarrow}\left(\begin{array}{lll}
0.41 & 0.37 & 0.22
\end{array}\right) .
$$

One of the uses for hospital planning based on the above result is, that a membership of a patient remains in hospital pathway 1, in the long run, almost surely 0.41 of the time the hospital is in operation. Another useful physical meaning for hospital planning, is that the relative population structure of the memberships in the various hospital pathways tends asymptotically almost surely to $\left(\begin{array}{lll}0.41 & 0.37 & 0.22\end{array}\right)$.

\subsection{A University System}

In this subsection we illustrate an application of a cyclic non-homogeneous Markov system in a University system. The importance of cyclic behavior was firstly stressed in Bartholomew (1982), p.71, where he also provided an interesting application of this concept which arose in Gani's (1963) study of student enrolment at Michigan State University. We consider the university system in Vassiliou and Tsantas (1984) with 3 years of study where the students that fail their year repeat it in the following year. The estimates of the transition probability matrices taken from Vassiliou and Tsantas (1984) assuming a cyclic repetition are

$$
\begin{gathered}
\mathbf{P}(3 t)=\left(\begin{array}{ccc}
0.17188 & 0.81875 & 0 \\
0 & 0.29873 & 0.68644 \\
0 & 0 & 0.32303
\end{array}\right), \\
\mathbf{P}(3 t+1)=\left(\begin{array}{ccc}
0.19379 & 0.7726 & 0 \\
0 & 0.33503 & 0.65482 \\
0 & 0 & 0.53563
\end{array}\right), \\
\mathbf{P}(3 t+2)=\left(\begin{array}{ccc}
0.16631 & 0.81641 & 0 \\
0 & 0.34318 & 0.64557 \\
0 & 0 & 0.50860
\end{array}\right) .
\end{gathered}
$$

Also

$$
\begin{gathered}
p_{4}(3 t)=\left(\begin{array}{lll}
0.00937 & 0.01483 & 0.67697
\end{array}\right), \\
p_{4}(3 t+1)=\left(\begin{array}{lll}
0.03361 & 0.01015 & 0.46437
\end{array}\right),
\end{gathered}
$$

and

$$
p_{4}(3 t+2)=\left(\begin{array}{lll}
0.01728 & 0.011250 .49140
\end{array}\right),
$$

and $\mathbf{p}_{0}(t)=\left(\begin{array}{lll}1 & 0 & 0\end{array}\right)$ for every $t=0,1,2, \ldots$. The total population of students is $T(0)=$ $8970, T(1)=9000, T(2)=9050$ and $T(t)=9050$ for $t=3,4, \ldots$. Then we have

$$
\begin{gathered}
\mathbf{Q}(3 t)=\left(\begin{array}{ccc}
0.181 & 0.819 & 0 \\
0.015 & 0.299 & 0.686 \\
0.677 & 0 & 0.323
\end{array}\right), \quad \mathbf{Q}(3 t+1)=\left(\begin{array}{ccc}
0.227 & 0.773 & 0 \\
0.010 & 0.335 & 0.655 \\
0.464 & 0 & 0.536
\end{array}\right), \\
\mathbf{Q}(3 t+2)=\left(\begin{array}{ccc}
0.184 & 0.816 & 0 \\
0.011 & 0.343 & 0.646 \\
0.491 & 0 & 0.509
\end{array}\right), \text { for } t=0,1,2, \ldots
\end{gathered}
$$


Then

$$
\begin{gathered}
\mathbf{Q}_{0}=\left(\begin{array}{lll}
0.277 & 0.182 & 0.541 \\
0.388 & 0.303 & 0.359 \\
0.147 & 0.427 & 0.426
\end{array}\right), \mathbf{Q}_{1}=\left(\begin{array}{lll}
0.354 & 0.176 & 0.470 \\
0.433 & 0.305 & 0.262 \\
0.253 & 0.399 & 0.348
\end{array}\right), \\
\mathbf{Q}_{2}=\left(\begin{array}{lll}
0.274 & 0.167 & 0.559 \\
0.308 & 0.381 & 0.311 \\
0.179 & 0.470 & 0.351
\end{array}\right) .
\end{gathered}
$$

Applying Eq. 2.3 recursively using the above data we get

$$
\begin{aligned}
& \mathbf{q}(0)=\left(\begin{array}{lll}
0.32 & 0.30 & 0.38
\end{array}\right), \quad \mathbb{E}[\mathbf{q}(0,3)]=\left(\begin{array}{lll}
0.242 & 0.315 & 0.443
\end{array}\right), \\
& \mathbb{E}[\mathbf{q}(0,6)]=\left(\begin{array}{lll}
0.238 & 0.329 & 0.433
\end{array}\right), \quad \mathbb{E}[\mathbf{q}(0,9)]=\left(\begin{array}{lll}
0.241 & 0.328 & 0.431
\end{array}\right), \\
& \mathbb{E}[\mathbf{q}(0,12)]=\left(\begin{array}{lll}
0.241 & 0.328 & 0.431
\end{array}\right) .
\end{aligned}
$$

We observe that $\mathbb{E}[\mathbf{q}(0,3 t)]$ converges. Since, $\Delta T(t)=0$ for $t=3,4, \ldots$ and $\mathbf{Q}_{0}$ is a regular stochastic matrix then the conditions of Theorem $13 \mathrm{~b}$ are satisfied and hence $\mathbf{q}_{0}(\infty)$ as given by Theorem $13 \mathrm{~b}$ should coincide with $\mathbb{E}[\mathbf{q}(0,3 t)]$. This was found to be true. The same was found with $\mathbb{E}[\mathbf{q}(0,3 t+1)] \rightarrow_{t \rightarrow \infty}(0.3400 .2950 .365)$, $\mathbb{E}[\mathbf{q}(0,3 t+1)] \rightarrow_{t \rightarrow \infty}(0.3400 .2950 .365)$, which were found equal with $\mathbf{q}_{1}(\infty)$ and $\mathbf{q}_{2}(\infty)$ given by Theorem $13 \mathrm{~b}$.

The Cezaro sum

$$
\frac{1}{t} \sum_{n=0}^{t-1} \mathbb{E}[0, n]
$$

was found to converge as early as $t=12$ to $(0.2780 .3310 .391)$. The two conditions of Theorem 14 are valid in this case since for the model used $\mathbf{Q}_{0}$ is regular and $\Delta T(t)=0$ for $t=3,4, \ldots$. According to Theorem 14 the strong run distribution was found to be equal with the limit of the Cezaro sum.

The conditions of Theorem 15 are satisfied for the present problem again since $\mathbf{Q}_{0}$ is regular and $\Delta T(t)=0$ for $t=3,4, \ldots$. In order to find $\boldsymbol{v}(t)$ and verify that

$$
\boldsymbol{v}(t) \stackrel{L^{2}}{\rightarrow} \frac{1}{d} \sum_{s=0}^{d-1} \mathbf{q}_{s}(\infty)
$$

it is equivalent according to relation (5.1) in the proof of Theorem 15 to verify that

$$
\lim _{t \rightarrow \infty} \mathbb{E}\left[\left(\boldsymbol{v}(t)-\frac{1}{d} \sum_{s=0}^{d-1} \mathbf{q}_{s}(\infty)\right)^{2}\right]=0 .
$$

For the present University System it was found that

$$
\lim _{t \rightarrow \infty} \mathbb{E}\left[\left(\boldsymbol{v}(t)-\left(\begin{array}{lll}
0.278 & 0.331 & 0.391
\end{array}\right)\right)^{2}\right]=0 .
$$

Hence,

$$
\boldsymbol{v}(t) \stackrel{L^{2}}{\rightarrow}\left(\begin{array}{llll}
0.278 & 0.331 & 0.391
\end{array}\right) \text { as } t \rightarrow \infty .
$$

Now, since $\mathbf{Q}_{0}$ is regular and $\Delta T(t)=0$ for $t=3,4, \ldots$, that is, the convergence is in geometric rate, the conditions of Theorem 17 are satisfied and thus

$$
\boldsymbol{v}(t) \stackrel{\text { a.s. }}{\rightarrow}(0.2780 .3310 .391) \text { as } t \rightarrow \infty .
$$

One of the uses for University planning physical meaning of the above result is, that a membership of a student place remaining in the first year of study, in the long run, almost surely 0.278 of the time the University is working. Another useful physical meaning for University 
planning, is that the relative population structure of the memberships in the various years of study tends asymptotically almost surely to $\left(\begin{array}{llll}0.278 & 0.331 & 0.391\end{array}\right)$.

Open Access This article is distributed under the terms of the Creative Commons Attribution 4.0 International License (http://creativecommons.org/licenses/by/4.0/), which permits unrestricted use, distribution, and reproduction in any medium, provided you give appropriate credit to the original author(s) and the source, provide a link to the Creative Commons license, and indicate if changes were made.

\section{References}

Bartholomew DJ (1963) A multistage renewal process. J R Stat Soc Soc B25:150-168

Bartholomew DJ (1967) Stochastic models for Social processes, 1st edn. Wiley, New York

Bartholomew DJ (1982) Stochastic models for Social processes, 3rd edn. Wiley, New York

Faddy M, McClean SI (2005) Markov chain modeling for geriartic patient care. Methods Archive 44(3):369-373

Foucher Y, Mathew E, Saint Pierre P, Durand J-F, Daures JP (2005) A semi-Markov model based on Weibull distribution with an illustration for HIV disease. Biometrical J 47(6):1-9

Gani J (1963) Formula for projecting enrollments and degrees awarded in Universities. J R Stat Soc A 126:400-409

Garg L, McClean SI, Meenan B, Millard P (2009) Non homogeneous Markov models for sequential pattern mining of health care data. IMA J Manag Math 20(4):327-344

Garg L, McClean SI, Meenan B, Millard P (2010) A non-homogeneous discrete time Markov model for admission scheduling and resource planning in a cost capacity constraint healthcare system. Health care Management Science 13(2):155-169

Georgiou AC, Vassiliou P-CG (1992) Periodicity of asymptotically attainable structures in nonhomogeneous Markov systems. Linear Algebra Appl 176:137-174

Georgiou AC, Tsantas N (1996) Non-stationary cyclic behavior in Markov systems. Linear Algebra Appl 237:549-587

Grimmett G, Stirzaker D (2001) Probability and random processes, 3rd edn. Oxford University Press, Oxford

Hall P, Heyde CC (1980) Martingale limit theorem and its applications. Academic Press, New York

Huang C, Isaacson DL, Vinograde B (1976) The rate of convergence of certain nonhomogeneous Markov chains. Z Wahrscheinlichkeitstheorie verw Gabiete 35:141-146

Isaacson DL, Madsen RW (1976) Markov Chains Theory and Applications. Wiley, New York

Kemeny JG, Snell JL (1981) Finite Markov chains. Springer, New York

Kemeny JG, Snell JL, Knapp AW (1976) Denumerable Markov Chains. Springer, New York

Mathiew E, Foucher Y, Dellamonica P, Daures J-P (2006) Parametric and non-homogeneous semi-Markov process for HIV control. Working paper Archer Hospital, Nice

Lalit G, McClean SI, Meenan B, Millard P (2010) A non-homogeneous discrete time Markov model for admission scheduling and resource planning in a cost or capacity constrained healthcare system. Health Care Management Science 13:155-169

Marshall AH, McClean SI, Shapcott CM, Millard P (2002) Modelling patient duration of stay to facilitate resource management of geriatric hospitals. Health Care Management Science 5:313-319

Marshall AH, McClean SI (2003) Conditional phase-type distributions for modelling patient length of stay in hospital. Int Trans Op Res 10:565-576

Marshall AH, McClean SI (2004) Using coxian Phase-Type distributions to identify patient characteristics for duration of stay in hospital. Health Care Management Science 7:285-289

McClean SI, Millard P (1998a) A three compartment model of the patient flows in a geriatric department: a decision support approach. Health Care Management Science 1:159-163

McClean SI, McAlea B, Millard P (1998b) Using a Markov reward model to estimate spend-down costs for geriatric department. J Oper Res Soc 49:1021-1025

McClean SI, Scotney B, Robinson S (2003) Conceptual clustering of heterogeneous gene expressions sequences. Artif Intell Rev 20:53-73

McClean SI, Millard P (2007) Where to treat the older patient? Can Markov models help us better understand the relationship between hospital and community care? J Oper Res Soc 58(2):255-261

McClean SI, Gillespie J, Garg L, Barton M, Scotney B, Fullerton K (2014a) Using phase-type models to cost stroke patient care across health, social and community services. Eur J Oper Res 236(1):190-199

McClean SI, Garg L, Fullerton K (2014b) Costing mixed Coxian phase-type systems with Poisson arrivals. Communications in Statistics-Theory and Methods 43(7):1437-1452 
Meyn S, Tweedie R (2009) Markov chains and stochastic stability. Cambridge University Press, Cambridge

Vadori N, Swishchuk A (2015) Strong Law of Large Numbers and Central Limit theorems for functionals of inhomogeneous Semi-Markov Processe. Stoch Anal Appl 33:213-243

Taylor GJ, McClean SI, Millard P (2000) Stochastic models of geriatric patient bed occupancy behaviour. J R Statist Soc A 163:Part 1, 39-48

Vassiliou P-CG (1982) Asymptotic behavior of Markov systems. J Appl Prob 19:851-857

Vassiliou P-CG (1984) Cyclic behavior and asymptotic stability of non-homogeneous Markov systems. J Appl Probab 21:315-325

Vassiliou P-CG (1986) Asymptotic variability of nonhomogeneous Markov systems under cyclic behavior. Eur J Oper Res 27:215-228

Vassiliou P-CG, Georgiou AC (1990) Asymptotically attainable structures in nonhomogeneous Markov systems. Oper Res 38(3):537-545

Vassiliou P-CG (1981) On the limiting behavior of a nonhomogeneous Markov chain model in manpower systems. Biometrika 68(2):557-561

Vassiliou P-CG, Tsantas N (1984) Maintainability of structures in nonhomogeneous Markov systems under cyclic behavior and input control. SIAM J Appl Math 44(5):1014-1022

Vassiliou P-CG (1997) The evolution of the theory of non-homogeneous Markov systems. Applied Stochastic Models Data Anal 13:159-176

Ugwuowo FI, McClean SI (2000) Modelling heterogeneity in a manpower system : a review. Appl Stoch Models Bus Ind 2:99-100

Young A, Almond G (1961) Predicting distributions of staff. Comp J 3:246-250 